\title{
EL ROL DE LA PERSONALIDAD EN LA RELACIÓN ENTRE FRUSTRACIÓN - ESTRÉS Y DIVERSAS REACCIONES AFECTIVAS: RESULTADOS DE UNA INVESTIGACIÓN
}

\author{
THE ROLE OF PERSONALITY IN THE RELATIONSHIP BETWEEN \\ STRESS - FRUSTRATION AND DIVERSE AFFECTIVE REACTIONS: \\ RESEARCH RESULTS
}

\author{
Soledad Cordero \\ Universidad Católica del Uruguay
}

\begin{abstract}
Resumen: En el presente artículo se presenta una investigación, cuyo objetivo fue estudiar y comparar la función mediadora de catorce estilos de personalidad en la relación entre el estrés y/o la frustración y el desarrollo de diversas reacciones afectivas. Se utilizaron dos muestras clínicas $(n=260)$ de pacientes adultos, con diversos síndromes clínicos (Eje I), asistidos ambulatoriamente en Alemania. El encuadre teórico de la investigación fue la Teoría de las Interacciones de los Sistemas de la Personalidad ${ }^{1}$, desarrollada por Julius Kuhl (Alemania). Los resultados obtenidos sugieren que los estilos de personalidad más vulnerables a desarrollar emociones negativas tras una frustración o bajo estrés son el estilo pasivo (depresivo $^{2}$ ) y el impulsivo (borderline).
\end{abstract}

Palabras claves: Estilos de personalidad, frustración, estrés, disposiciones afectiva, Teoría PSI

\begin{abstract}
The aim of the study was to investigate the mediation role of fourteen personality styles in the relationship between stress and/or frustration and the development of a variety of affective reactions. We used two clinical samples $(n=260)$ of adult outpatients with diverse clinical syndromes (Axis I). The theoretical framework was Kuhl's Personality Systems Interaction Theory (Germany). The results suggest that the personality styles that are more vulnerable to develop negative emotions - in a state of frustration or under stress - are the passive (depressive) and the impulsive (borderline) personality styles.
\end{abstract}

Key words: personality styles, frustration, stress, affective reactions, PSI Theory

\section{INTRODUCCIÓN}

El presente trabajo tiene como objetivo explorar la influencia de las variables situacionales (frustración y estrés) y de diferentes estilos de personalidad $(E P)^{3}$ sobre una variedad de reacciones afectivas (afecto negativo y su inhibición, afecto positivo y su inhibición). Se parte de la base que tanto la frustración de las necesidades sociales (necesidad de logro, de afiliación y de poder), así como distintos tipo de even- tos estresantes, pueden aumentar el riesgo de desarrollar emociones negativas (Emmons, 1986; Brunstein, 2001, Holmes \& Rahe, 1967; Scully, Tosi \& Banning, 2000). Además se parte de la premisa de que el impacto de la frustración de las necesidades básicas y del estrés sobre el individuo está influido por el estilo de personalidad. En relación con este punto existe un gran número de investigaciones que han explorado diversas variables aisladas de la personalidad que podrían actuar como

\footnotetext{
1 Teoría PSI por sus siglas en alemán (Persönlichkeits-System-InteraktionsTheorie)

${ }^{2}$ Entre paréntesis los trastornos de personalidad a los que corresponden cada estilo de personalidad mencionado. ${ }^{3}$ Para facilitar la lectura se utiliza la abreviación EP para los estilos de personalidad.

Esta investigación forma parte de una Tesis de doctorado, realizada en el Departamento de Psicologìa Diferencial e Investigación de la Personalidad de la Universidad de Osnabrück, financiada por el Servicio Alemàn de Intercambio Estudiantil (DAAD).

Correspondencia: Prof. Dra. Soledad Cordero, Facultad de Psicologìa, Universidad Catòlica del Uruguay: e-mail: scordero@ucu.edu.uy
} 
moduladoras o reductoras del estrés. El objetivo de dichas investigaciones ha sido comprender y explicar qué es lo que distingue a las personas que afrontan eficientemente las adversidades y mantienen un buen ajuste psicológico de las que no lo consiguen (Ramírez Maestre, Esteve y López, 2001). Dentro de las variables de personalidad estudiadas se encuentran la autoestima, autoeficacia, optimismo y locus de control, personalidad robusta (Kosaba, 1979), sentido de coherencia (Antonosvsky, 1979), ilusiones positivas (Taylor, 1991) o personalidad resistente (Major, Richards, Cooper, Cozzarelli y Zubek, 1998) (Ramírez Maestre et al., 2001). Existen además un gran número de estudios con el modelo de los cinco factores, que señalan al neuroticismo como el predictor más potente del afecto negativo.

Lo cierto es que cada vez han ido surgiendo posiciones más cercanas a la concepción de la personalidad como mediadora de la relación entre estrés y bienestar personal (Sánchez Cánovas y Sánchez López, 1994)

El trabajo presentado en este artículo se centra específicamente en el examen de hipótesis de mediación: ¿qué estilos de personalidad son más vulnerables a desarrollar estados emocionales negativos cuando se encuentran bajo estrés o tras una frustración? La función mediadora representa según Baron y Kenny (1986) "el mecanismo generador a través del cual la variable independiente es capaz de influir en las variables dependientes de interés". Se comparan las funciones mediadoras de catorce EP (entendidos, en base a un modelo dimensional, como variantes normales de los trastornos de personalidad del DSM-IV) en la relación entre frustración/estrés y una variedad de reacciones afectivas. Adicionalmente se evaluó el posible rol mediacional de la interacción entre los estilos de personalidad y algunas variables de autorregulación (Orientación al estado; Kuhl, 1983).

Si bien hay trabajos en los cuales se analiza la relación entre algunas de las variables a estudiar, no se encuentran investigaciones que relacionen todas las variables consideradas en el presente trabajo, por lo que se lo concibió como un estudio de tipo exploratorio. Esta investigación forma parte de un trabajo más extenso en el cual se examinó además el rol de los EP para el desarrollo de un amplio espectro de síntomas clínicos (Eje I) (Cordero, 2005, 2007).

El encuadre teórico de la investigación es la Teoría de las Interacciones de los Sistemas de la Personalidad (Teoría PSI), que fue desarrollada por el Prof. Dr. Julius Kuhl (Director del Departamento de Psicología Diferencial e Investigación en Personalidad, Universidad de Osnabrück, Alemania). Los trabajos iniciales de Kuhl se plasmaron en la Teoría del control de la acción (1983), cuyo objetivo es el estudio de los principales problemas volitivos (inicio de la acción, persistencia y superación de obstáculos). La teoría PSI (Kuhl, 1998, 2000a, 2001) es una ampliación de la Teoría del Control de la Acción, en la cual, se elabora de manera más detallada el concepto del "self" (en español: el yo, no obstante, en este trabajo se utilizará el término en inglés), y su rol dentro del Control de la Acción. La teoría aborda además otras temáticas, dentro de las cuales se encuentran los trastornos de la personalidad. A continuación se describen las premisas básicas y conceptos más relevantes de dicha teoría.

\section{MARCo TEÓRICO: LA TEORÍa de LAS INTERACCIONES DE LOS SISTEMAS DE LA PERSONALIDAD}

La teoría PSI es un enfoque sistémico y analítico funcional que explica la personalidad a través de la interacción entre diversos sistemas psíquicos. Dentro de sus características más destacables se encuentran: el carácter integrativo (toma conceptos y aportes de la psicología de la personalidad, de la motivación, las ciencias cognitivas neurológicas y la psicología clínica), el análisis del interjuego entre emoción y cognición, y el apoyo empírico del modelo.

La teoría PSI gira en torno a tres premisas básicas (Kuhl, 2001): a) la personalidad se basa en disposiciones afectivas; 2 ) la motivación depende del tipo de respuesta afectiva; 3 ) la personalidad es una configuración de sistemas.

La primera premisa considera que la combinación de afectos positivo y negativo ayuda a explicar fenómenos que tienen que ver con la personalidad y sus diferentes estilos. Autores como Eysenck (1990) y Gray (1987) han relacionado las diferencias de la personalidad con la sensibilidad hacia los afectos positivo y negativo. Gran cantidad de hallazgos de la psicología del desarrollo y de la personalidad sugieren que existen diferencias individuales en la sensibilidad a los afectos positivo y negativo, las cuales son en parte genéticas y en parte debidas a las experiencias de la primera infancia (Kuhl, 2001). 
Cabe aclarar en este punto que Kuhl (2001) utiliza el concepto de afecto haciendo referencia más a sus características funcionales (ej. inhibición o facilitación de la conducta) que a las vivencias que implica. Por disposición afectiva se entiende la tendencia a presentar más frecuentemente una determinada emoción ( $\mathrm{y}$ a sentirla de forma más duradera). Por otra parte la emoción hace referencia a un concepto general que incluye dentro de sí una serie de subcategorías tales como sentimientos y estados de ánimo (emociones de más larga duración).

La segunda premisa considera que las necesidades humanas y la forma en que se llevan a cabo las metas se relacionan directamente con los motivos sociales (Murray, 1943) y sobre todo, con los motivos de afiliación, de logro y de poder.

La tercera premisa considera que la personalidad es el resultado de la interacción dinámica (más o menos estable) entre diferentes niveles o sistemas. La teoría PSI postula siete niveles de funcionamiento de la personalidad: (1) operaciones cognitivas elementales (identificación de objetos y control del comportamiento intuitivo); (2) temperamento (activación motora y "arousal" sensorial); (3) afectos positivo y negativo; (4) enfrentamiento "coping" emocional (modos regresivo y progresivo); (5) motivos (basados en el conocimiento autobiográfico sobre cómo satisfacer las necesidades de afiliación, poder, logro en diversos contextos); (6) operaciones cognitivas de orden superior (pensamiento analítico y planificación intencional vs. procesamiento holístico y experiencia autobiográfica); y (7) autorregulación - autocontrol centrado en las metas vs. autorregulación creativa (Kuhl, 2001).

\section{Los estilos y trastornos de la personali- dad según la teoria PSI}

La teoría PSI propone un modelo dimensional de los estilos de personalidad, en el cual, en contraste con el modelo categorial (orientado hacia la presencia o ausencia de un trastorno), las características de personalidad son entendidas como dentro de un continuo que va de la normalidad a la anormalidad. Los trastornos de la personalidad son vistos como formas extremas de estilos de personalidad adaptativos (Kuhl, 2001). De esto se deriva, que es la "intensidad" del estilo y no la "cualidad", lo que provoca problemas. Esta concepción de los estilos y trastornos de la personalidad dentro de un continuo es sostenida por otros autores como
Millon (1985), Schmitz y cols. (2001) y Oldham (1990).

Los estilos y trastornos se entienden en la teoría PSI como variantes estables vs. crónicas de configuraciones de sistemas (patrones de interacción entre los diferentes sistemas de la personalidad). Estas configuraciones son más que la suma de las características de cada subsistema.

La teoría diferencia catorce estilos y trastornos de la personalidad, que se corresponde sustancialmente con la clasificación de los trastornos de personalidad del DSM-IV (1994), a excepción de dos de ellos que no se encuentran dentro de dicho manual (el estilo voluntarioso/trastorno altruista de la personalidad y el estilo optimista/trastorno rapsódico de la personalidad). Una descripción de estos dos EP se encuentra en Cordero (2007).

Los estilos y trastornos de personalidad son explicados a través de patrones de interacción entre disposiciones afectivas y cognitivas.

En lo que respecta a las disposiciones afectivas, a cada trastorno de la personalidad se le asigna una combinación específica de disposiciones altas, medias o bajas hacia el afecto negativo y positivo. Por ejemplo el trastorno evitativo de la personalidad se caracteriza de acuerdo a la teoría PSI por una combinación de una alta sensibilidad para el afecto negativo y una baja sensibilidad para el afecto positivo $[A-\& A(+)]$. La tabla 1 resume las combinaciones planteadas por la teoría PSI para cada estilo de personalidad y su correspondiente trastorno (Kuhl, 2000a, 2001; Kuhl \& Kazén, 1997).

Un supuesto importante de la teoría es que las disposiciones afectivas no describen el estado de ánimo básico, sino formas de reaccionar frente al estrés y las demandas de la vida cotidiana. Las distintas disposiciones afectivas predestinan a diferentes formas de afrontar las emociones (Kuhl, 2001).

En cuanto a las disposiciones cognitivas se proponen cuatro sistemas cognitivos hipotéticos (denominados macrosistemas cognitivos): la memoria de extensión (ME), la memoria de intención (MI), el sistema de control intuitivo de la conducta $(\mathrm{CIC})$ y el sistema de identificación de objetos (IO). Cada uno de ellos se corresponde con las formas de procesar la información planteadas por Jung (1936/1990): el pensamiento se corresponde con la Ml, el sentimiento con la 
ME, la intuición con el CIC y la sensación con el IO. Kuhl desarrolla además las bases neuroanatómicas de los estilos cognitivos propuestos por Jung. La tabla 2 resume las características principales de cada uno de los macrosistemas cognitivos, que se apoyan en investigaciones experimentales (Kuhl, 1998) y en conocimientos aportados por las ciencias cognitivas y las neurociencias. Para una descripción más detallada de cada macrosistema cognitivo ver Cordero (2007).

Los macrosistemas cognitivos cumplen funciones relevantes para la personalidad y se asocian con procesos específicos de autogobierno (Kuhl, 1998). La ME es descrita como la base cognitiva del "self" (la autopercepción de sí mismo, las representaciones implícitas sobre las propias necesidades, valores, afectos y eventos de vida). El comportamiento guiado por la ME (self) se caracteriza por la autodeterminación (autocongruencia), el apoyo emocional (automotivación), la creatividad y la flexibilidad en la resolución de problemas.

La teoría postula un antagonismo entre el primer par de macrosistemas cognitivos ( $\mathrm{Ml}$ y $\mathrm{CCl}$ ), los cuales regulan la ejecución real de las metas propuestas y el segundo par de sistemas (ME e IO) que tienen como propósito regular el aprendizaje, desarrollo y cambio de la personalidad a través de la integración de la experiencia.

Las características de cada uno de los macrosistemas cognitivos se aplican a la interpretación de los distintos estilos y trastornos de la personalidad. De acuerdo a la función cognitiva predominante una persona puede ser más intuitiva, más perceptiva (o sensitiva), más sentimental, o más racional. Los estilos de personalidad se caracterizan por la relativa dominancia de uno o más sistemas cognitivos (y su correspondiente forma de procesar la información), mientras que en los trastornos de personalidad dominan crónicamente ciertas disposiciones cognitivas (ver Tabla 1).

Tabla 1. Hipótesis de la teoría PSI sobre las disposiciones cognitivas (los macrosistemas y sus respectivas formas de procesar la información) dominantes y subordinadas para cada uno de los trastornos de la personalidad y sobre la sensibilidad de hacia el afecto positivo y negativo. Los estilos que aparecen en esta tabla son los que mide el Inventario de estilos y trastornos de la personalidad (PSSI).

\begin{tabular}{|c|c|c|c|c|c|c|}
\hline $\begin{array}{c}\text { Estilo } \\
\text { de personalidad }\end{array}$ & $\begin{array}{c}\text { Trastorno } \\
\text { de } \\
\text { personalidad }\end{array}$ & $\begin{array}{c}\text { Funciones } \\
\text { cognitivas } \\
\text { dominantes }\end{array}$ & $\begin{array}{c}\text { Funciones } \\
\text { cognitivas } \\
\text { subordinadas }\end{array}$ & \multicolumn{3}{|c|}{$\begin{array}{l}\text { Sensibilidad hacia el } \\
\text { afecto positivo y negativo }\end{array}$} \\
\hline Autodeterminado & Antisocial & $\mathrm{S}$ & $P$ & $A\{+\}$ & $\&$ & A (-) \\
\hline Desconfiado & Paranoide & $P, S$ & SS & $A(+)$ & $\&$ & $A(-)$ \\
\hline Reservado & Esquizoide & $P$ & S, SS & A (+) & $\&$ & $A\{-\}$ \\
\hline Inseguro & Evitativo & $P, S S$ & S & $A(+)$ & $\&$ & A - \\
\hline Esmerado & Obsesivo & SS & $S$ & $A\{+\}$ & $\&$ & A - \\
\hline Idiosincrático & Esquizotípico & SS, I & $P, S$ & $A+$ & \& & A - \\
\hline Optimista & Rapsódico & I & S, SS & $A+$ & $\&$ & $A\{-\}$ \\
\hline Ambicioso & Narcisista & S, I & SS, P & $A+$ & $\&$ & A (-) \\
\hline Crítico & Negativista & $P, S$ & I, SS & $A(+)$ & $\&$ & A (-) \\
\hline Leal & Dependiente & SS, P & S, I & $A(+)$ & \& & A \\
\hline Impulsivo & Borderline & SS, I & $P, S$ & $A+$ & $\&$ & A - \\
\hline Amable & Histriónico & I & P & $A+$ & $\&$ & $A\{-\}$ \\
\hline Pasivo & Depresivo & $P, S S$ & I, S & $A(+)$ & \& & A - \\
\hline Voluntarioso & Altruista & SS I & $P, S$ & A $(+)$ & $\&$ & A \\
\hline
\end{tabular}

Nota: P (pensamiento, MI), S (sentimiento; ME), I (intuición, CIC), SS (sensación, IO)

Las sensibilidades hacia los afectos se describen con los siguientes símbolos:

$A+$ : fuerte sensibilidad hacia el afecto positivo; $A\{+\}$ : sensibilidad media hacia el afecto positivo; $A(+)$ : baja sensibilidad hacia el afecto positivo (tendencia a inhibir el afecto positivo); A-: fuerte sensibilidad hacia el afecto negativo; A $\{-\}$ : sensibilidad media hacia el afecto negativo; A (-): baja sensibilidad hacia el afecto negativo (tendencia a inhibir el afecto negativo). 
Tabla 2. Características de los cuatro macrosistemas cognitivos propuestos en la teoría PSI (Kuhl, 2001)

\begin{tabular}{|c|c|c|}
\hline Nivel de agregación & Hemisferio izquierdo & Hemisferio derecho \\
\hline Complejo (prefrontal) & 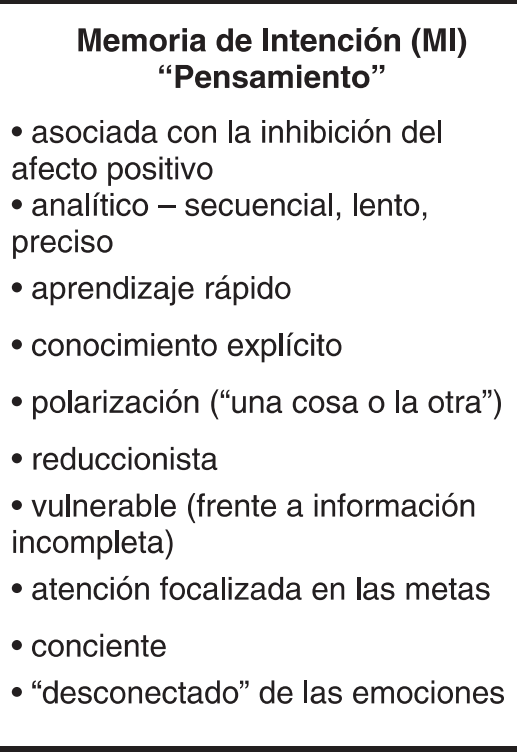 & $\begin{array}{l}\text { Memoria de Extensión (ME) } \\
\text { “Sentimiento” / Base cognitiva del } \\
\text { SELF } \\
\text { - asociada con la inhibición del afecto } \\
\text { negativo } \\
\text { - holístico - paralelo, rápido, } \\
\text { impresionista } \\
\text { - aprendizaje lento } \\
\text { - conocimiento implícito } \\
\text { - integración de contradicciones } \\
\text { - sensible a las diferencias } \\
\text { - robusto (frente a información } \\
\text { incompleta) } \\
\text { - atención orientada a las congruencias } \\
\text { - no conciente } \\
\text { - Percepción y regulación de las } \\
\text { emociones: acceso al self }\end{array}$ \\
\hline Elemental (parietal) & $\begin{array}{l}\text { Identificación de objetos } \\
\text { "Sensación" } \\
\text { - asociado con el afecto negativo } \\
\text { - analítico, secuencial, lento, } \\
\text { específico } \\
\text { - orientado al pasado } \\
\text { - reconocimiento } \\
\text { - vulnerable } \\
\text { - atención orientada a las } \\
\text { incongruencias } \\
\text { - conciente }\end{array}$ & $\begin{array}{l}\text { Control intuitivo de la conducta } \\
\text { "Intuición" } \\
\text { - asociado con el afecto positivo } \\
\text { - holístico, paralelo, rápido, } \\
\text { impresionista } \\
\text { - orientado al presente y al futuro } \\
\text { - anticipación } \\
\text { - robusto } \\
\text { - atención espacial } \\
\text { - no conciente }\end{array}$ \\
\hline
\end{tabular}

\section{Hipótesis de modulación afectiva}

En la teoría PSI se analiza la influencia de los afectos sobre los procesos cognitivos y el procesamiento de la información. Específicamente se postulan una serie de hipótesis acerca de la forma en que los afectos positivos y negativos modulan la activación de los sistemas cognitivos y su comunicación (Kuhl, 2000a, 2001). A continuación se describen brevemente las dos hipótesis más importantes de modulación afectiva propuestas en la teoría PSI.

1era hipótesis de modulación afectiva: Describe los efectos del afecto positivo y su inhibición sobre la relación entre la memoria de intención (MI) y el sistema de control intuitivo de la conducta $(\mathrm{CIC})$ : el afecto positivo $[\mathrm{A}+]$ ayuda a convertir las intenciones explicitas en acciones, mientras que la inhibición del afecto po- sitivo $[\mathrm{A}(+)]$ acentúa la influencia del pensamiento analítico y la planificación (MI), facilitando el mantenimiento cognitivo de las intenciones en la Ml. Este proceso es adaptativo cuando es necesario resolver un problema o es necesario esperar para llevar a cabo una meta. Posteriormente cuando un estímulo positivo es generado externa o internamente (automotivación), la inhibición del afecto positivo es abolida y se llevan a cabo las intenciones a través de la activación del CIC. En otras palabras la primera modulación afectiva describe como el afecto positivo $[\mathrm{A}+]$ atenúa o inhibe la influencia del pensamiento analítico y la planificación (MI) e intensifica la conducta intuitiva $(\mathrm{CIC})$ y como la inhibición del afecto positivo $[\mathrm{A}(+)]$ tiene un efecto inverso.

2da hipótesis de modulación afectiva: Describe los efectos del afecto negativo y su 
inhibición sobre la relación entre el self (ME) y el sistema de percepción de discrepancias (IO). El afecto negativo [A - ] inhibe la influencia de la ME (y por lo tanto también de las representaciones integradas del self) sobre las acciones y percepciones de la persona e intensifica la influencia del sistema de percepción de discrepancias (IO), poniendo de relieve todo aquello que se desvía de las expectativas del sujeto. La inhibición del self dificulta la percepción global de las situaciones así como la utilización de las funciones creativas en la resolución de problemas. Cuando la percepción de sí mismo se bloquea (inhibición del self) la persona se ve alienada de sus propios sentimientos y preferencias, pudiendo confundir los deseos y expectativas ajenas con las propias (Kuhl \& Kazén, 1994). Inversamente la regulación del afecto negativo $[A(-)]$ estimula el acceso al self e inhibe el IO, permitiendo que las nuevas percepciones sean integradas en el self. Por otra parte la activación del self tiene un rol importante en la autorregulación del afecto, como lo demuestran diversas investigaciones (Koole \& Jostmann, 2003; Linville, 1987; Showers \& Kling, 1996).

Las hipótesis de modulación afectiva permiten deducir, en base a las disposiciones afectivas de una persona, sus sistemas cognitivos dominantes o subordinados. Así por ejemplo una fuerte disposición a reaccionar con afecto positivo (ej. en el trastorno histriónico de la personalidad) se relaciona con un debilitamiento del pensamiento analítico (MI) y un predominio del sistema de control intuitivo de la conducta $(\mathrm{ClC})$. La sensibilidad para el afecto negativo (ej. en el trastorno evitativo de la personalidad) debilita la percepción global de las situaciones, que es proporcionada por la memoria de extensión (y el self), y estimula el sistema de identificación de discrepancias (tendencia a focalizarse en sensaciones conflictivas e incongruentes, y en aspectos aislados, como por ejemplo la mirada crítica de otra persona).

\section{El modelo de la estrella de los estilos y trastornos de la personalidad}

Sobre la base de la teoría PSI se desarrollo el modelo de la estrella (Fig. 1) de los estilos y trastornos de la personalidad (Kuhl, 2001; Kuhl \& Kazén, 1997). El mismo muestra una „proyección" de los estilos y los trastornos de la personalidad propuestos en la teoría PSI sobre las dos dimensiones motivacionales básicas, es decir sobre la relativa manifestación de la activación de los sistemas de recompensa y castigo y sus correspondientes afectos negativos y positivos. El modelo de la estrella muestra las diferentes combinaciones posibles ("configuraciones de sistemas") derivadas de la manifestación de los afectos positivos y negativos. La disposición de los estilos y trastornos en el modelo de la estrella tiene el estatus de hipótesis, de ahí que se hable de un modelo y no de una teoría.

En el eje horizontal de la estrella se encuentran las dimensiones del afecto positivo (a la izquierda las disposiciones bajas [A (+)], a la derecha las disposiciones fuertes $[A+]$ ) y en el vertical las del afecto negativo (arriba las disposiciones bajas [A (-)], abajo las disposiciones fuertes [A-]). Las dos dimensiones afectivas se encuentran dispuestas en forma ortogonal, ya que son concebidas como dimensiones separadas, que pueden variar en forma independiente. Estudios previos sugieren que el afecto positivo y negativo son dimensiones independientes (Watson y Tellegen, 1985).

Cada punta de la estrella se corresponde con una determinada configuración de sistemas (y de esa forma con un estilo de personalidad), que se caracteriza por un patrón específico de interacción de las funciones cognitivas y afectivas. El medio de la estrella no se corresponde con ningún estilo, sino con la capacidad para moverse en forma flexible dentro de las ocho configuraciones de sistemas (puntas de la estrella). Una persona con una personalidad flexible puede "moverse” en diversas direcciones a partir del centro de la estrella. En el medio de la estrella se encuentran las configuraciones de sistemas que son concebidas como de corta duración, como estrategias de resolución de problemas, que pueden estabilizarse o rigidizarse a medida que se alejan del medio de la estrella. El modelo de la estrella simboliza de este modo la concepción dimensional de los estilos y trastornos de la personalidad. Los estilos de personalidad se transforman, según la teoría, en trastornos, cuando se produce una "fijación" en una determinada configuración de sistemas. En términos del modelo de la estrella esto significa que una persona se encuentra en una punta de la estrella y le es muy difícil "moverse" de ella, a pesar de que una situación así lo requiera. Las posibilidades de acción están en ese caso limitadas. Así, por ejemplo, una persona esquizoide no podrá manejar su estilo analítico, aún cuando se encuentre en una si- 
tuación que requiera un intercambio emocional y cercanía afectiva.

El polo de afecto positivo $[A+]$ de la estrella está representado por el trastorno histriónico de la personalidad. Dentro de este polo se encuentran también los tipos "optimista-rapsódico" y "ambicioso/narcisista". Todos estos estilos tienen en común el estar relacionados con una fuerte sensibilidad para el afecto positivo, lo que de acuerdo a la 1era modulación afectiva conduce a una conducta que va desde la espontaneidad a la impulsividad. Al final de este polo [A $(+)$ ] se ubican los estilos que están relacionados con una baja sensibilidad para el afecto positivo (inhibición del afecto positivo), lo que de acuerdo a la 1era modulación afectiva puede llevar a una actitud reflexiva y a una pasividad depresiva (en este último caso si hay una disposición extrema hacia la inhibición del afecto positivo).

En el polo de afecto negativo [A -] se encuentran estilos que se caracterizan por una baja seguridad en sí mismos y una tendencia a reaccionar con temor y ansiedad. Son estilos con una alta sensibilidad para el afecto negativo, lo que de acuerdo a la 2da modulación afectiva conduce a dificultades en el acceso al self. Estos EP pueden mostrar una fuerte capacidad de acción, pero esta siempre esta orientada a la realización de las metas ajenas. Aquí se encuentran ubicados trastornos tales como el evitativo y el dependiente. El extremo opuesto de este polo [A (-)] se caracteriza por el contrario por la fuerte autonomía y autodeterminación. Aquí se encuentran trastornos tales como el antisocial, narcisista, paranoide y negativista, que comparten una fuerte tendencia a inhibir el afecto negativo. Dentro del modelo de la estrella se encuentran también los denominados "estilos mezclados", que se ubican en una posición intermedia (ej. negativista) y que se caracterizan por la combinación de una baja sensibilidad para el afecto positivo y para el afecto negativo $[A(+) \& A(-)]$.

En suma, la teoría PSI postula que cada estilo y trastorno se vincula con un patrón específico de interacción entre las disposiciones afectivas (sensibilidad para el afecto positivo y

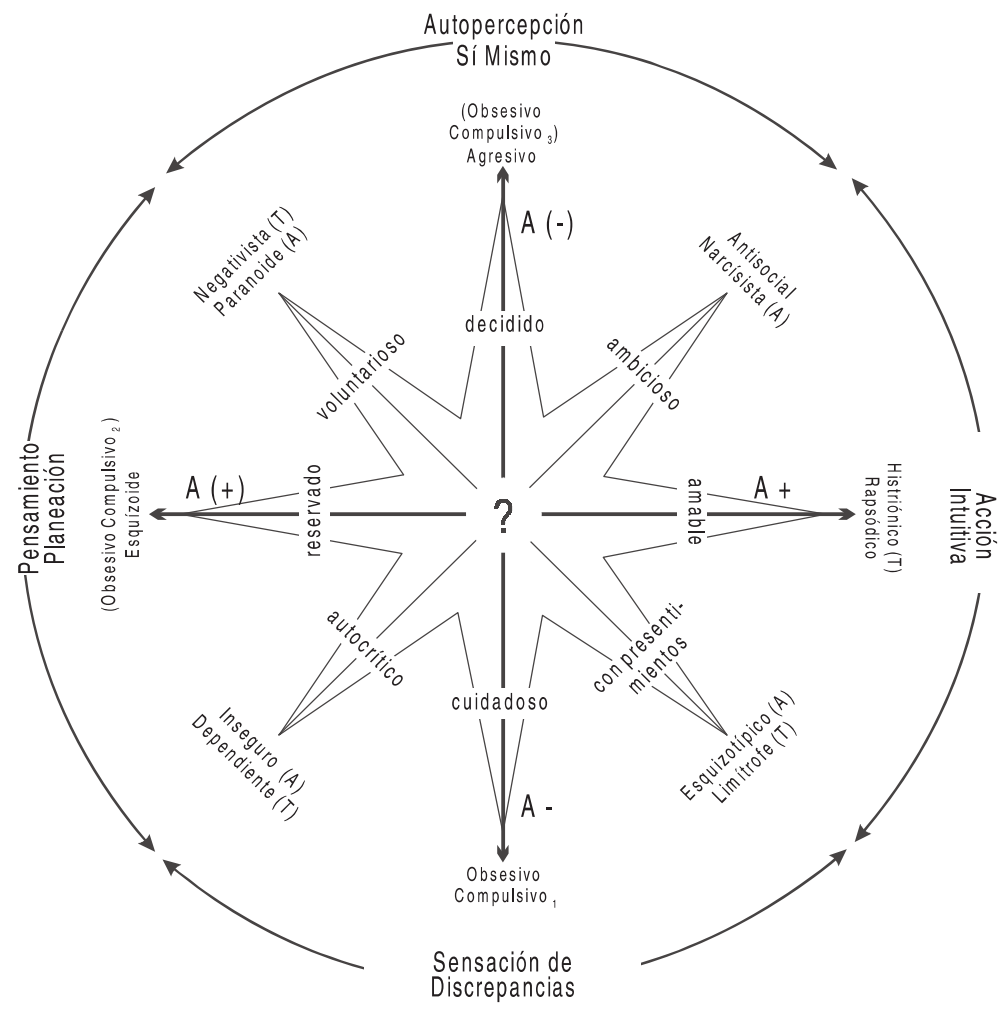

Fig. 1. El modelo STAR (estrella) de los estilos (circulo interno) y de los trastornos de la personalidad (circulo externo).

Nota: Autopercepción de sí mismo se refiere al self y la memoria de extensión (ME, sentir), acción intuitiva con el sistema de control intuitivo de la conducta ( $\mathrm{CIC}$, intuir), pensamiento y planeación con la memoria de intención (MI, pensar) y sensación de discrepancias con el sistema de identificación de objetos (IO, sensación). 
negativo) y las disposiciones cognitivas (dominancia de alguno de los cuatro macrosistemas cognitivos postulados en la teoría PSI y sus respectivas formas de procesar la información: pensar, sentir, intuir y percibir) (Kuhl, 1995).

\section{Primera y segunda reacción afectiva}

La teoría PSI se diferencia dos tipos de reacciones afectivas. La primera se refiere a la disposición (alta, media o baja) que tiene una persona a reaccionar con afecto positivo o negativo. La segunda describe la capacidad de autorregulación afectiva, es decir la capacidad que tiene una persona para autorregular un estado afectivo específico una vez este lo ha invadido. Los EP son entendidos en términos de la primera respuesta afectiva. Para la teoría la segunda reacción afectiva es más importante para el afrontamiento del estrés y de las emociones negativas que la primera: una fuerte sensibilidad al afecto negativo no conduce necesariamente a problemas psíquicos, cuando este puede ser regulado en forma adecuada y rápida (Biebrich \& Kuhl, 2002a). Una buena capacidad de autorregulación significa que la persona ha desarrollado la capacidad para regular por sí mismo los estados afectivos. Estas personas tienen un riesgo considerablemente más bajo de enfermarse (Hautzinger, 1994). Por otro lado, una baja sensibilidad (por ejemplo en los extrovertidos) no significa siempre también estabilidad.

Esta afirmación ha sido comprobada empíricamente. En una investigación de Biebrich y Kuhl (2002b), se observo que personas con una puntuación alta en la escala de neuroticismo podían manejar adecuadamente el afecto negativo cuando poseían además buenas capacidades de autorregulación y acceso al self (esto significa que podían confrontarse directamente con sus emociones), mientras que en las personas con una baja puntuación en neuroticismo el afecto negativo era regulado en mayor medida a través de estrategias de evitación. Desde el punto de vista teórico, estos hallazgos son explicados de la siguiente forma: la persona con una fuerte sensibilidad puede aprender siempre cosas nuevas del mundo interno o externo, y a su vez - en el caso de tener una buena autorregulación - puede afrontar adecuadamente las experiencias dolorosas e integrarlas en el self, contribuyendo así a su desarrollo (Kuhl, 2001, Pág. 991). Se trata de un equilibro entre la sensibilidad para el afecto negativo y la capacidad para afrontar activamente las experiencias dolorosas.
La autorregulación se relaciona con procesos como la capacidad de regulación de los afectos, las estrategias de afrontamiento, la capacidad de resolución de problemas, el autocontrol, la capacidad para llevar a cabo las propias metas a pesar de las dificultades internas o externas (Kuhl, 1994), etc. Es especialmente importante en los casos en que la sensibilidad para el afecto negativo es alta y/o la sensibilidad para el afecto positivo es baja.

\section{Autorregulación: Orientación al estado y orientación a la acción}

Las diferencias individuales en la capacidad de autorregular los afectos bajo estrés son descritas a través de los constructos de orientación al estado versus orientación a la acción. Las personas con una orientación al estado (OE) están predispuestas a experimentar, tras un fracaso o tras vivencias aversivas, pensamientos indeseados y no controlables acerca de la situación (preocupación) y a vacilar a la hora de llevar a cabo sus intenciones (Kuhl, 1994). Las personas con orientación a la acción (OA) son en cambio capaces de liberarse de pensamientos indeseados acerca de la situación aversiva y de llevar a cabo sus intenciones o metas de forma autogobernada (iniciativa).

La orientación a la acción es entendida como la capacidad para regular por sí mismo los afectos positivo y negativo (Kuhl \& Beckmann, 1994). Las personas orientadas a la acción regulan sus emociones (ej. miedo) por sí mismas, mientras que las orientadas al estado presentan dificultades en este sentido.

A su vez dentro de cada una de las disposiciones (OE y OA) se diferencian dos subtipos de acuerdo a la capacidad de autorregulación afectiva:

a) Orientación a la acción tras un fracaso $(O A F)$ : es concebida como la capacidad para reducir ("downregulate") por sí mismo el afecto negativo una vez que este ha aparecido (autorelajarse).

b) Orientación a la acción prospectiva (OAP): es concebida como la habilidad para autogenerar ("upregulate") el afecto positivo con el fin de afrontar dificultades (automotivarse), dado que el afecto positivo tiene un efecto de facilitación conductual y volitiva (Kuhl \& Kazén, 1999).

c) Orientación al estado prospectiva (OEP): se define como la dificultad para recuperar el afecto positivo perdido (por ej. debido a una si- 
tuación difícil o frustrante) sin el apoyo externo. Estas personas tienen dificultades para automotivarse y se caracterizan por presentar un déficit en la energía, que impide la puesta en práctica de las intenciones.

d) Orientación al estado tras un fracaso (OEF): se define como la dificultad para regular el afecto negativo (autorelajarse) sin apoyo externo.

Un gran número de investigaciones apoyan la conceptualización de la orientación al estado versus orientación a la acción en términos de la habilidad para autorregular el afecto (Brunstein, 2001; Koole \& Jostmann, 2003; Kuhl \& Beckmann, 1994). Por ejemplo, Brunstein (2001) encontró una disociación entre la sensibilidad afectiva (neuroticismo) y la regulación afectiva (OAP y OAF) en estudiantes durante el transcurso de un semestre. Mientras que el neuroticismo correlacionaba de forma positiva y significativa con el afecto negativo al principio del semestre, la orientación a la acción tras un fracaso (OAF) se asociaba con una reducción significativa del afecto negativo durante el transcurso del semestre. De forma similar, la orientación a la acción prospectiva (OAP) se asociaba significativamente con un aumento del afecto positivo durante el transcurso del semestre. Estos resultados son consistentes con la afirmación de Kuhl (2001) de que los constructos afectivos clásicos como neuroticismo y extraversión miden la primera reacción afectiva, mientras que los constructos de orientación al estado y a la acción se relacionan con la segunda reacción afectiva.

Una capacidad de adaptación óptima supone la capacidad para controlar los estados emocionales de acuerdo a la situación. Las funciones de autorregulación no dependen de acuerdo a la teoría PSI solamente de aspectos disposicionales, sino también de las características de la situación concreta, en especial de las situaciones estresantes con las que la persona debe confrontarse. En la teoría los acontecimientos estresantes son separados en: (1) acontecimientos de vida amenazantes (ej. peligros, experiencias dolorosas, situaciones impredecibles, la incertidumbre) que se asocian con un aumento del afecto negativo y requieren de la habilidad para regular el afecto negativo (OAF) y (2) acontecimientos de vida demandantes (ej. conflicto con las metas, tareas de gran dificultad y obstáculos) que se asocian con una disminución del afecto positivo y requieren de la habilidad para recuperar el afecto positivo (OAP). Varios hallazgos apoyan la interacción asumida entre OAF y acontecimientos amenazantes y entre OAP y acontecimientos demandantes (Kuhl \& Beckmann, 1994). En la investigación que se presenta a continuación se midieron ambos tipos de estresores.

En una investigación llevada a cabo por Baumann, Kaschel y Kuhl (2004) se examino el efecto de interacción entre la primera y segunda reacción afectiva (estilos de personalidad y competencias de autorregulación). En concordancia con lo esperado, se encontraron interacciones específicas entre un estilo de personalidad y un tipo de orientación a la acción con respecto al bienestar emocional y una variedad de síntomas psíquicos. Las personas con una personalidad fuertemente esquizoide mostraban un alto riesgo de desarrollar síntomas cuando su capacidad para automotivarse era muy débil (OEP) y las personas con una personalidad fuertemente evitativa cuando su capacidad para autorelajarse era muy débil (OEF). En la teoría se plantea como hipótesis que los déficit en la autorregulación juegan un papel fundamental en el desarrollo de los trastorno de la personalidad, en otras palabras una fuerte disposición afectiva (estilos de personalidad) puede volverse patológica, cuando esta reducida la capacidad de autorregulación de los sentimientos, pensamientos y acciones.

\section{HiPótesis}

Fueron formuladas en el marco de la teoría PSI. Se formularon las siguientes hipótesis generales: (1) Las disposiciones afectivas que de acuerdo a la teoría PSI subyacen a los EP influyen en que estos tengan o no una función mediadora en las relaciones a explorar; (2) los EP

Tabla 3. Estadística descriptiva de la muestras

\begin{tabular}{lcccccccc}
\hline & & \multicolumn{2}{c}{ Sexo } & & \multicolumn{3}{c}{ Edad } \\
\cline { 3 - 4 } \cline { 7 - 8 } & $N$ & Mujeres & Hombres & & Media & Min. & Max. \\
\hline Muestra 1 & 126 & $70(56 \%)$ & $56(44 \%)$ & & 40,6 & 17 & 71 \\
\hline Muestra 2 & 137 & $62(45 \%)$ & $75(55 \%)$ & & 37,9 & 16 & 64 \\
\hline
\end{tabular}


con una alta sensibilidad para el afecto negativo (evitativo, obsesivo, esquizotípico, dependiente, borderline, depresivo y altruista) y/o una baja sensibilidad para el afecto positivo (paranoide, esquizoide, evitativo, negativista, dependiente y depresivo) tendrán una función mediadora en las relaciones a explorar; (3) los EP con una baja sensibilidad para el afecto negativo y/o una alta sensibilidad para el afecto positivo (antisocial, narcisista, rapsódico e histriónico) no tendrán una función mediadora o la tendrán en menor medida que los anteriores; (4) la función mediadora de cada uno los EP variará de acuerdo al predictor (frustración, demandas o presión) y (5) de acuerdo al tipo de afecto analizado. No se realizaron hipótesis respecto a cada uno de los estilos de personalidad por separado.

\section{MÉTodo}

\section{Muestra}

Se utilizaron dos muestras clínicas ( $\mathrm{n}$ total = 260) de pacientes adultos con diversos síndromes clínicos (Eje I) asistidos ambulatoriamente en varías clínicas en Alemania. Los criterios de exclusión para este estudio fueron los diagnósticos de esquizofrenia, psicosis actual o trastorno bipolar agudo.

\section{Procedimiento e instrumentos de medida}

Los participantes completaron los cuestionarios luego de la primera entrevista con el psiquiatra, como parte de la evaluación general y antes de iniciar el tratamiento.

Todos los datos fueron recabados a través de cuestionarios, los cuales corresponden a la batería de diagnóstico analítico-funcional de la personalidad (TOP ${ }^{4}$, Autor: J. Kuhl y cols., Alemania). A continuación se describen las variables introducidas en los análisis y los cuestionarios a los que corresponden:

Estilos de personalidad: Fueron evaluados a través del Inventario de estilos y trastornos de la personalidad (PSSI; Kuhl \& Kazén, Alemania, 1997). Este inventario autoadministrado, está orientado según los criterios del DSM-IV y del ICD-10 y mide, basándose en una concepción dimensional, estilos no patológicos (ver tabla 1) correspondientes a los diversos trastor- nos de la personalidad clasificados en dichos manuales. Consta de 140 ítems, que se ordenan en 14 escalas. El sujeto debe valorar, a través de una escala de Likert de "nada" (0) a "totalmente" (4), hasta que punto se aplican a su persona una serie de afirmaciones. Las escalas del inventario han demostrado tener suficiente consistencia interna en diversos estudios con población clínica y no-clínica (Kuhl, 2001; Kuhl \& Kazén, 1997).

Estrés: El estrés fue medido a través de dos subescalas (amenazas/presión y demandas), de 4 ítems cada una de ellas, pertenecientes a la versión abreviada del Inventario de Autogobierno (SSI; Kuhl \& Fuhrmann, 1998). EI SSI mide las diversas competencias del autogobierno (ej. autorregulación, autocontrol) así como la inhibición de dichas competencias bajo situaciones de estrés o frustración. Se compone de 48 ítems. El sujeto debe valorar hasta que punto se aplican a su persona una serie de afirmaciones, en una escala de Likert de "nada" (0) a "totalmente" (4). Un ejemplo de la variable demandas es: "Actualmente el trabajo (o el estudio) se me hace muy pesado" y de la variable amenazas (o presión): "Actualmente tengo que afrontar muchas experiencias dolorosas en mi vida".

Frustración de las necesidades sociales: Como medida global de la frustración se utilizo una escala del Test de Implementación de Motivos (MUT; Kuhl, 1999). Este test mide las representaciones concientes de las necesidades de afiliación, logro y poder y la forma de su implementación (intuitiva, con perspectiva, estratégica, pasiva). La escala utilizada evalúa la anticipación de resultados negativos e incluye ítems asociados con las tres necesidades sociales básicas (afiliación, logro y poder). Ejemplos de ítems de esta escala son: „Cuando alguien me rechaza, me siento como si estuviera paralizado“ (motivo de afiliación), „Los fracasos por lo general me quitan completamente el ánimo" (motivo de logro), "Frente a personas que se muestran muy seguras me siento frecuentemente inferior" (motivo de poder).

Dimensiones afectivas: fueron medidas a través del Inventario de Emociones Básicas (BEF-3). Este inventario autoadministrado consiste en una lista de adjetivos que permiten medir la intensidad de 7 factores afectivos (incluye ítems de Watson, Clark, \& Tellegen, 1988, y

\footnotetext{
${ }^{4}$ La versión original del TOP fue desarrollada en alemán. Actualmente existen traducciones al español de los inventarios que la componen.
} 
descriptores afectivos adicionales). Se compone de 21 ítems, que se ordenan en 7 subescalas, cada una de las cuales consta de 3 ítems que se valoran en una escala de Likert de 0 (nada) a 3 (completamente). Los sujetos reciben la siguiente instrucción: "La mayoría de las veces me siento...". Los 7 factores afectivos se encuentran ordenados en las siguientes categorías: (1) alegría (mide el afecto positivo); (2) tristeza (mide el afecto negativo); (3) activación (motora, mide un tipo positivo de activación); (4) excitación (en inglés "arousal", mide un tipo de activación sensorial negativa); (5) desánimo/inhibición (mide la inhibición del afecto positivo); (6) serenidad (inhibición del afecto negativo) e (7) irritación.

Autorregulación: La orientación a la acción y al estado (OA y OE) fue evaluada a través de un cuestionario denominado Cuestionario del Control de la Acción (HAKEMP-90; Kuhl, 1994). Este cuestionario contiene 2 escalas: 1) Orientación a la acción subsecuente al fracaso (OAF) y 2) Orientación a la acción prospectiva (OAP). Cada escala contiene 12 reactivos que describen diferentes situaciones. Para cada situación existen dos alternativas de respuesta, una de ellas indica la orientación a la acción y la otra la orientación al estado. Un ejemplo de un ítem de la escala OAF: "Cuando me dicen que mi trabajo ha sido completamente insatisfactorio: a) no dejo que eso me preocupe durante mucho tiempo, o b) me deja paralizado". La opción a refleja la OAF y la b la OEF. Un ejemplo de un ítem de la escala OAP: "Cuando sé que tengo que terminar algo pronto: a) Frecuentemente tengo que obligarme para empezar, o b) Me resulta fácil ponerme a hacerlo y terminarlo". La opción a refleja la OEP y la b la OAP. EI HAKEMP-90 tiene suficiente confiabilidad (coeficientes Alfa de Cronbach > .70) y una validez de constructo adecuado (Kuhl \& Beckmann, 1994). La distinción teórica de los componentes de la escala de orientación a la acción en OAP y OAF fueron confirmados a través de la estructura factorial (Dieffendorf, Hall, Lord, \& Strean, 2000; Kuhl \& Beckmann, 1994).

\section{Análisis de los datos}

Para el análisis estadístico de los datos se utilizó el programa informático SPSS 12.0. Para responder a las preguntas planteadas se examinaron varios modelos de mediación, a través de análisis de regresión múltiple (siguiendo el procedimiento recomendado por Baron y Kenny (1986) para los análisis de mediación).

Para que un modelo mediacional pueda considerarse válido deben cumplirse, de acuerdo a estos autores, los siguientes pasos: (1) La variable independiente o predictiva, debe tener una influencia directa (significativa) sobre la variable dependiente o criterio (este paso corresponde a la línea a de la Fig. 2); (2) Debe existir una relación significativa entre la variable predictiva y el mediador (línea b); (3) La influencia del mediador sobre la variable dependiente debe ser significativa (línea c); (4) La influencia directa del predictor sobre la variable dependiente debe dejar de ser significativa, cuando se con-

Fig. 2: Resumen de todas las variables introducidas en los modelos de mediación evaluados

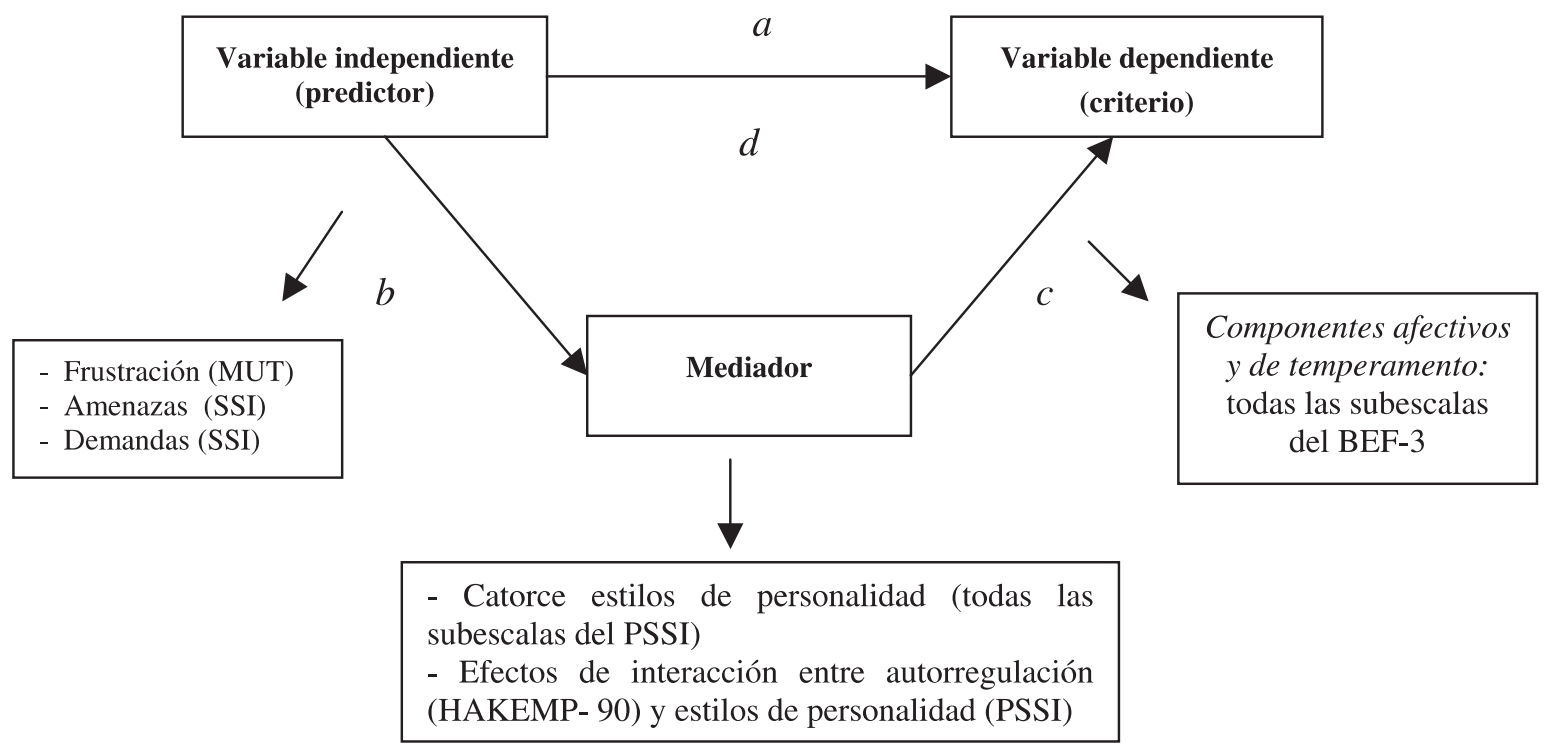


trola la influencia del mediador, es decir cuando se introduce el mediador en el análisis (línea $d$ ) y (5) el resultado del Test de Sobel (Sobel Test, 1982, ver Baron \& Kenny, 1986) para comprobar el vinculo mediacional indirecto debe ser significativo.

Todas las variables fueron estandarizadas antes de realizar los cálculos. Dado que los afectos explorados pueden responder a diversas causas se contemplo a los EP como mediadores parciales. Todos los análisis de regresión se calcularon para cada una de las dos muestras por separado, con el objetivo de analizar el grado en que los resultados de una muestra se replicaban en la otra.

Cada una de las variables predictoras (frustración y los dos factores de estrés) se introdujo por separado en los análisis de regresión. El mismo procedimiento se siguió con las varia- bles mediadoras (cada uno de los estilos de personalidad) y las variables dependientes (diversas reacciones afectivas).

Adicionalmente se introdujo como variable mediadora la interacción de cada uno de los EP con las escalas OAF y OAP invertidas (es decir orientación al estado tras un fracaso (OEF) y orientación al estado prospectiva (OEP). Estos análisis se calcularon únicamente para la variable frustración como predictora.

\section{Resultados}

En primer lugar, y con el fin de analizar las características de ambas muestras, se compararon las diferencias de medias (por medio del estadístico $\mathrm{T}$ para muestras independientes) para todas las variables a explorar. Los análisis realizados indican que las dos muestras se di-

Tabla 3. Comparación de las puntuaciones medias en las escalas del BEF-3

\begin{tabular}{|l|c|c|c|c|c|}
\cline { 2 - 5 } \multicolumn{1}{c|}{} & \multicolumn{2}{c|}{ Muestra 1 } & \multicolumn{2}{c|}{ Muestra 2 } & Media \\
\hline Escalas del BEF-3 & Media & DT & DT & $\begin{array}{c}\text { Estadístico } \\
\text { T }\end{array}$ \\
\hline Tristeza A - & 43,96 & 10,90 & 39,31 & 11,95 &, $001^{* *}$ \\
\hline Desánimo A (+) & 46,58 & 14,95 & 40,86 & 19,17 &, $008^{* *}$ \\
\hline Alegría A + & 48,12 & 11,40 & 41,22 & 13,36 &, $000^{* * *}$ \\
\hline Serenidad A (-) & 55,60 & 14,49 & 60,32 & 16,23 &, $014^{*}$ \\
\hline Activación T+ & 53,61 & 11,89 & 58,99 & 12,40 &, $000^{* * *}$ \\
\hline Excitación T - & 55,08 & 12,10 & 59,94 & 15,184 &, $005^{* *}$ \\
\hline Irritación & 54,78 & 13,91 & 56,51 & 13,89 &, $316 \mathrm{~ns}$ \\
\hline
\end{tabular}

Tabla 4. Comparación de las puntuaciones medias en las escalas del Inventario de Estilos y Trastornos de la personalidad (PSSI)

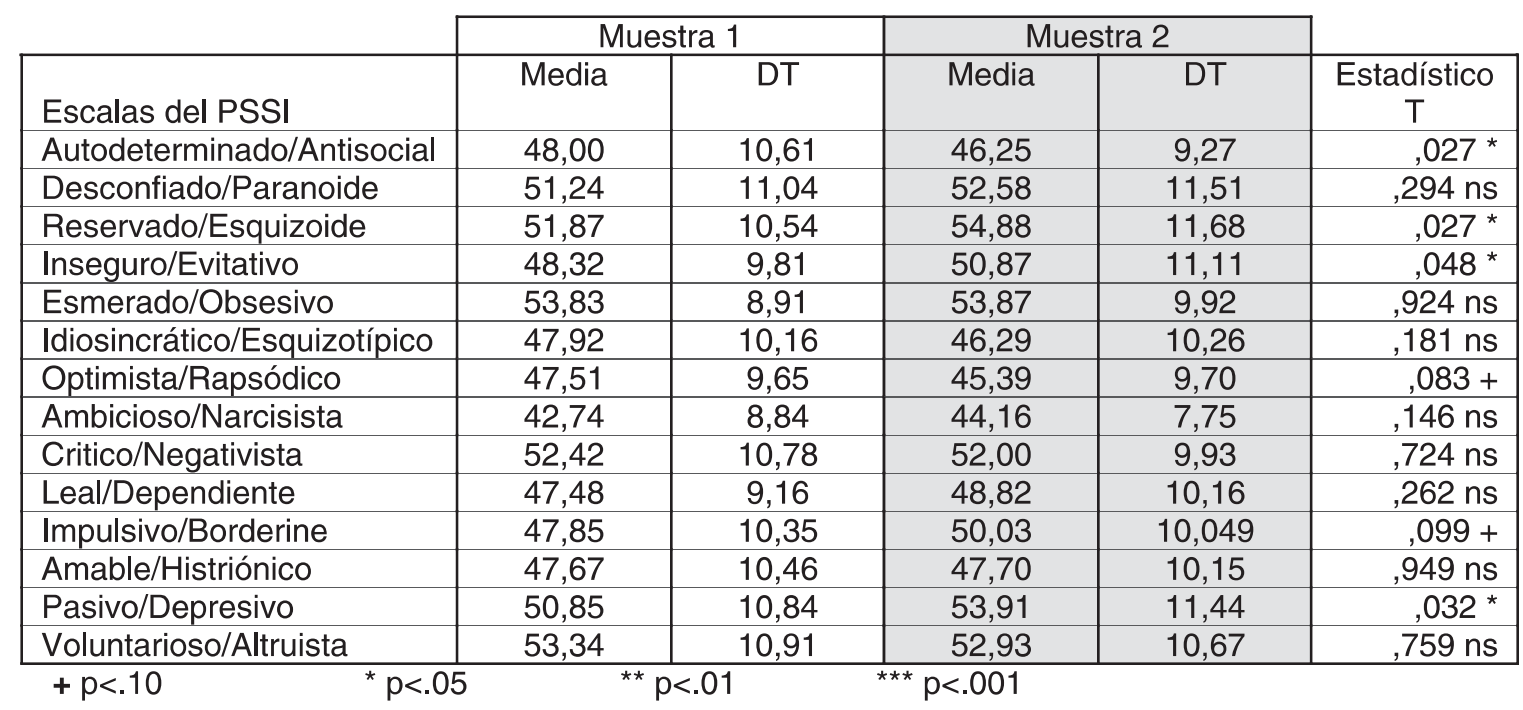


ferencian significativamente entre sí en las escalas del BEF-3 (Tabla 3).

Los pacientes de la muestra 2 tienen una puntuación significativamente más alta en tristeza, desánimo y excitación que los pacientes de la muestra 1 y una puntuación significativamente más baja en serenidad, alegría y activación.

En la tabla 4 se muestran las medias de ambas muestras en las subescalas del PSSI. Los pacientes de la muestra 2 tienen puntuaciones significativamente más altas en las escalas reservado/esquizoide, inseguro/evitativo y pasivo/ depresivo que las personas de la muestra 1. Una diferencia marginalmente significativa se observa en la escala impulsivo/borderline. No aparecen diferencias significativas entre ambas muestras en el resto de las variables exploradas (frustración, demandas y amenazas).

Se utilizaron correlaciones de Pearson para evaluar las relaciones entre todas las variables analizadas. Estos análisis se realizaron para cada una de las muestras por separado. Debido al gran número de correlaciones calculadas se describen a continuación los resultados más importantes.

Muestra 1. En ambas muestras los índices de correlación obtenidos indican que las tres variables predictoras (la frustración y los dos variables de estrés) se asocian positiva y significativamente con las escalas tristeza, excitación, desánimo e irritabilidad, y negativa y significativamente con las escalas alegría, acti- vación y serenidad. Es importante señalar en este punto que no se encontró un patrón diferencial de correlaciones entre las dos variables de estrés. En otros estudios realizados (Kuhl, 2001, Pág. 243) estas subescalas del PSSI mostraron una correlación teóricamente consistente con un bajo nivel de afecto positivo, en el caso de la variable demandas, y con un alto nivel de afecto negativo, en el caso de la escala amenazas.

Todos EP, que de acuerdo a la teoría PSI se asocian con una baja sensibilidad para el afecto positivo (paranoide, esquizoide, evitativo, negativista, dependiente y depresivo) y/o una alta sensibilidad para el afecto negativo (evitativo, obsesivo, esquizotípico, dependiente, borderline, depresivo y altruista) correlacionaron en general negativa y significativamente con las escalas de alegría, activación y serenidad (BEF-3). A su vez estos estilos se asocian positivamente con las escalas que miden tristeza, excitación, desánimo e irritación. Por otro lado, las escalas del PSSI que miden los EP asociados con una baja sensibilidad para el afecto negativo y/o una alta sensibilidad para el afecto positivo (antisocial, narcisista, rapsódico e histriónico) obtienen un perfil de correlaciones inverso al de los EP anteriormente mencionados. En líneas generales el patrón de correlaciones obtenido es consistente con las hipótesis planteadas por la teoría PSI respecto a las disposiciones afectivas de los estilos y trastornos de personalidad.

Además se calcularon las correlaciones en-

Tabla 5. Resumen de los resultados que se replican en ambas muestras

\begin{tabular}{|l|l|l|l|}
\hline $\begin{array}{l}\text { Subescalas del BEF-3 } \\
\text { (variables dependientes) }\end{array}$ & $\begin{array}{c}\text { La variable } \\
\text { frustración como } \\
\text { predictora }\end{array}$ & $\begin{array}{c}\text { La variable } \\
\text { demandas } \\
\text { como predictora }\end{array}$ & $\begin{array}{c}\text { La variable amenazas } \\
\text { como predictora }\end{array}$ \\
\hline $\begin{array}{l}\text { Tristeza A - } \\
\text { Desánimo A (+) }\end{array}$ & $\mathrm{DP}$ & $\mathrm{DP}$ & -- \\
\hline $\begin{array}{l}\text { Alegría A+ } \\
\text { Serenidad A (-) }\end{array}$ & $\mathrm{DP}, \mathrm{BL}(R)$ & $\mathrm{DP}, \mathrm{BL}$ \\
\hline Activación T+ & $\mathrm{DP}(R)$ & $\mathrm{DP}$ & \\
\hline Excitación T - & $\mathrm{DP}$ & & $\mathrm{DP}, \mathrm{BL}, \mathrm{SU}, \mathrm{PN}(\mathrm{R})$ \\
\hline Irritación & $\mathrm{DP}, \mathrm{BL}$ & $\mathrm{DP}, \mathrm{BL}, \mathrm{NT}$ \\
\hline
\end{tabular}

Notas:

Abreviaciones de las escalas del PSSI: PN (desconfiado/paranoide), SU (autocrítico/evitativo), NT (crítico/ negativista), BL (espontáneo/borderline), DP (pasivo/depresivo)

$R$ (inverso): El mediador va con puntuaciones bajas en la correspondiente escala del BEF-3. Ej. DP y BL correlacionan negativamente serenidad. Es decir son mediadores para la relación entre frustración y una disminución de la serenidad.

- En las dos muestras ningún estilo de personalidad obtiene una función mediadora significativa para la relación entre el correspondiente predictor y la correspondiente subescala del BEF-3. 
tre las escalas del PSSI y las escalas del HAKEMP (OAP y OAF). En ambas muestras la escala OAF correlaciona negativa y significativamente con la escala de frustración (MUT): a mayor orientación a la acción, menor tendencia a la frustración. Los mismos resultados se obtienen para la escala OAP.

Todos los estilos y trastornos de personalidad, que de acuerdo a la teoría PSI se asocian con una baja sensibilidad para el afecto positivo (paranoide, esquizoide, evitativo, negativista, dependiente y depresivo) correlacionan en general negativa y significativamente con la escala OAP, es decir se relacionan con una tendencia a la orientación al estado prospectiva. Las escalas paranoide, esquizoide, evitativo, dependiente, borderline, depresivo y altruista correlacionan en ambas muestras negativa y significativamente con la escala OAF, es decir se relacionan positivamente con la tendencia a reaccionar tras un fracaso con una orientación al estado. Las escalas antisocial, rapsódico e histriónico se relacionan en ambas muestras positivamente con la escala OAF.

\section{Análisis de regresión}

Todos los resultados que se presentan a continuación se obtuvieron en ambas muestras (es decir se replicaron). Debido al gran número de variables analizadas y de análisis de regresión calculados se presentan aquí exclusivamente los resultados más importantes. La tabla 5 muestra de forma resumida los resultados de todos los análisis de regresión realizados.

A continuación se presentan los resultados del primer paso de los análisis de regresión (línea $a$ y $b$ Fig. 2) para cada uno de los predictores explorados (frustración, demandas y presión). El objetivo de este primer paso es analizar la influencia directa de cada una de las variables predictoras exploradas (frustración, demandas y presión) sobre las variables dependientes (subescalas del BEF-3) (Tablas $6,7$ y 8$)$

Como puede observarse en las tablas $(7,8 \mathrm{y}$ 9) las variables frustración, demandas y amenazas predicen las mismas reacciones afectivas: tristeza, excitación, desánimo, irritabilidad y una reducción de la activación, la alegría y la serenidad.

Tabla 6. Coeficientes de regresión $\beta$ (Beta) de los análisis de regresión con la variable frustración (MUT) como predictora y las escalas del BEF-3 como variables dependientes (criterio).

\begin{tabular}{|c|c|c|c|c|}
\hline \multirow[b]{2}{*}{ Variable dependiente (criterio) } & \multicolumn{2}{|c|}{ Muestra 1} & \multicolumn{2}{|c|}{ Muestra 2} \\
\hline & Beta & $\mathbf{T}$ & Beta & $\mathbf{T}$ \\
\hline Tristeza A - &, $48 * * *$ & 6,11 &, $51 * * *$ & 6,82 \\
\hline Desánimo A (+) &, $56 * * *$ & 7,43 &, $63 * * *$ & 9,31 \\
\hline Alegría A + &,$- 36 * * *$ & $-4,23$ &,$- 38 * * *$ & $-4,70$ \\
\hline Serenidad A (-) &,$- 35 * * *$ & $-4,10$ &,$- 43 * * *$ & $-5,51$ \\
\hline Activación T+ &,$- 32 * * *$ & $-3,78$ &,$- 42 * * *$ & $-5,38$ \\
\hline Excitación T - &, $43 * * *$ & 5,27 &, $54 * * *$ & 7,50 \\
\hline Irritación &, $35 * * *$ & 4,17 &, $35 * * *$ & 4,34 \\
\hline
\end{tabular}

Tabla 7. Coeficientes de regresión $\beta$ (Beta) de los análisis de regresión con la variable demandas (SSI) como predictora y las escalas del BEF-3 como variables dependientes (criterio).

\begin{tabular}{|c|c|c|c|c|}
\hline \multirow[b]{2}{*}{ Variable dependiente (criterio) } & \multicolumn{2}{|c|}{ Muestra 1} & \multicolumn{2}{|c|}{ Muestra 2} \\
\hline & Beta & $\mathbf{T}$ & Beta & $\mathbf{T}$ \\
\hline Tristeza A - &, $57^{\star \star \star}$ & 7,71 &, $51^{\star \star \star}$ & 6,77 \\
\hline Desánimo A (+) & , 42 *** & 5.14 &, 36 *** & 4,53 \\
\hline Alegría A + &,- 38 *** & $-4,54$ &,- 46 *** & $-5,99$ \\
\hline Serenidad A (-) &,$- 49^{\star \star \star}$ & $-6,27$ &,$- 44^{\star \star \star}$ & $-5,65$ \\
\hline Activación T+ &,$- 30^{* *}$ & $-3,51$ &,- 29 ** & $-3,53$ \\
\hline Excitación T - &, $53^{* \star *}$ & 6,85 &, $55^{* * *}$ & 7,69 \\
\hline Irritación &, $37^{* \star \star}$ & 4,35 & $31^{\star \star \star}$ & 3,81 \\
\hline
\end{tabular}


Tabla 8. Coeficientes de regresión $\beta$ (Beta) de los análisis de regresión con la variable amenazas (SSI) como predictora y las escalas del BEF-3 como variables dependientes (criterio).

\begin{tabular}{|l|r|r|r|r|}
\cline { 2 - 5 } \multicolumn{1}{c|}{} & \multicolumn{3}{c|}{ Muestra 1 } & \multicolumn{2}{c|}{ Muestra 2 } \\
\hline Tristeza A - & \multicolumn{2}{c|}{ Beta } & \multicolumn{1}{c|}{ T } & \multicolumn{1}{c|}{ Beta } \\
Desánimo A (+) &, $54^{* * *}$ & 7,05 &, $42^{* * *}$ & 5,35 \\
Alegría A + &, $38^{* * *}$ & 4,57 &, $34^{* * *}$ & 4,19 \\
Serenidad A (-) &,$- 36^{* * *}$ & $-4,30$ &,$- 43^{* * *}$ & $-5,53$ \\
Activación T+ &,$- 40^{* * *}$ & $-4,88$ &,$- 41^{* * *}$ & $-5,23$ \\
Excitación T - &,$- 26^{* *}$ & $-2,99$ &,$- 26^{* *}$ & $-3,08$ \\
Irritación &, $49^{* * *}$ & 6,23 &, $53^{* * *}$ & 7,17 \\
\hline
\end{tabular}

Tabla 9. Coeficientes de regresión $\beta$ (Beta) de los análisis de regresión con la variable Frustración (MUT) como predictora y las escalas del PSSI como variables dependientes (mediadores).

\begin{tabular}{|c|c|c|c|c|}
\hline \multirow[b]{2}{*}{ Variables dependientes (Mediator) } & \multicolumn{2}{|c|}{ Muestra 1} & \multicolumn{2}{|c|}{ Muestra 2} \\
\hline & Beta & $\mathbf{T}$ & Beta & $\mathbf{T}$ \\
\hline Autodeterminado/Antisocial &,$- 25^{\star \star}$ & $-2,91$ &,$- 16+$ & $-1,90$ \\
\hline Desconfiado/ Paranoide &, $32^{\star * \star}$ & 3,78 &, $37^{\star \star \star}$ & 4,58 \\
\hline Reservado/Esquizoide &, $32^{\star \star \star}$ & 3,73 &, $49^{\star \star \star}$ & 5,83 \\
\hline Esmerado/Obsesivo & ,10 ns & 1,10 & ,05 ns &, 62 \\
\hline Idiosincrásico/Esquizotípico & ,11 ns & 1,27 & ,06 ns &, 71 \\
\hline Optimista/Rapsódico &,$- 26^{\star *}$ & $-2,93$ &,$- 23^{* \star}$ & $-2,77$ \\
\hline Amable/Histriónico &,$- 24^{\star *}$ & $-2,73$ &,$- 35^{\star \star \star}$ & $-4,40$ \\
\hline Ambicioso/Narcisista &, $21^{*}$ & 2,36 &, $00 \mathrm{~ns}$ & ,02 \\
\hline Leal/Dependiente &, $42^{\star \star \star}$ & 5,07 &, $43^{\star \star \star}$ & 5,50 \\
\hline Impulsivo/Borderline &, $57^{\star \star \star}$ & 7,60 &, $71^{\star \star \star}$ & 11,63 \\
\hline Voluntarioso/Altruista &, $42^{\star \star *}$ & 5,07 &, $43^{\star * *}$ & 5,46 \\
\hline Crítico/Negativista &, $54^{\star \star \star}$ & 7,18 &, $48^{\star \star \star}$ & 6,23 \\
\hline Pasivo/Depresivo & $62^{\star \star \star}$ & 8,87 &, $76^{\star \star \star}$ & 13,66 \\
\hline Inseguro/Evitativo &, $73^{\star * \star}$ & 11,86 &, $78^{\star \star \star}$ & 14,59 \\
\hline
\end{tabular}

Tabla 10. Coeficientes de regresión $\beta$ (Beta) de los análisis de regresión con la variable Demandas (SSI) como predictora y las escalas del PSSI como variables dependientes (mediadores).

\begin{tabular}{|c|c|c|c|c|}
\hline \multirow[b]{2}{*}{ Variable dependiente (Mediador) } & \multicolumn{2}{|c|}{ Muestra 1} & \multicolumn{2}{|c|}{ Muestra 2} \\
\hline & Beta & $\mathbf{T}$ & Beta & $\mathbf{T}$ \\
\hline Autodeterminado/Antisocial &,$- 05 \mathrm{~ns}$ &,- 55 &,$- 04 \mathrm{~ns}$ &,- 48 \\
\hline Desconfiado/ Paranoide &, $32^{\star \star \star}$ & 3,79 &, $43^{\star \star \star}$ & 5,60 \\
\hline Reservado/Esquizoide &, $32^{\star \star \star}$ & 3,78 &, $30^{\star \star \star}$ & 3,67 \\
\hline Esmerado/Obsesivo &, $02 \mathrm{~ns}$ & ,27 &, $14 \mathrm{~ns}$ & 1,60 \\
\hline Idiosincrásico/Esquizotípico &, $26^{\star \star}$ & 3,00 &, $18^{*}$ & 2,17 \\
\hline Optimista/Rapsódico &,$- 16+$ & $-1,75$ &,$- 18^{\star}$ & $-2,07$ \\
\hline Amable/Histriónico &,$- 10 \mathrm{~ns}$ & $-1,08$ &,$- 11 \mathrm{~ns}$ & $-1,26$ \\
\hline Ambicioso/Narcisista & ,22* & 2,42 &, $11 \mathrm{~ns}$ & 1,29 \\
\hline Leal/Dependiente &, $36^{\star \star \star}$ & 4,31 &, $26^{\star \star}$ & 3,17 \\
\hline Impulsivo/Borderline &, $52^{\star \star \star}$ & 6,71 &, $45^{\star \star \star}$ & 5,92 \\
\hline Voluntarioso/Altruista &, $26^{\star *}$ & 2,10 &, $42^{\star * \star}$ & 5,42 \\
\hline Crítico/Negativista &, $65^{\star \star \star}$ & 9,50 &, $60^{\star \star \star}$ & 8,70 \\
\hline Pasivo/Depresivo &, $59^{\star \star \star}$ & 8,13 &, $48^{\star \star \star}$ & 6,40 \\
\hline Inseguro/Evitativo &, $37^{\star \star \star}$ & 4,37 & $40^{\star \star \star}$ & 5,07 \\
\hline
\end{tabular}


Tabla 11. Coeficientes de regresión $\beta$ (Beta) de los análisis de regresión con la variable presión (SSI) como predictora y las escalas del PSSI como variables dependientes (mediadores).

\begin{tabular}{|c|c|c|c|c|}
\hline \multirow[b]{2}{*}{ Variable dependiente (Mediador) } & \multicolumn{2}{|c|}{ Muestra 1} & \multicolumn{2}{|c|}{ Muestra 2} \\
\hline & Beta & $\mathbf{T}$ & Beta & $\mathbf{T}$ \\
\hline Autodeterminado/Antisocial & ,02 ns & .18 &,$- 03 \mathrm{~ns}$ &,- 30 \\
\hline Desconfiado/ Paranoide &, $38^{* * *}$ & 4,59 &,$- 46^{\star \star \star}$ & 6,08 \\
\hline Reservado/Esquizoide & $28^{\star *}$ & 3,02 &, $18^{\star}$ & 2,06 \\
\hline Esmerado/Obsesivo &, $07 \mathrm{~ns}$ &, 74 & ,13 ns & 1,57 \\
\hline Idiosincrásico/Esquizotípico & $24^{* *}$ & 2,74 & $29^{\star *}$ & 3,56 \\
\hline Optimista/Rapsódico &, $10 \mathrm{~ns}$ & $-1,13$ &,$- 16+$ & $-1,86$ \\
\hline Amable/Histriónico &,$- 08 \mathrm{~ns}$ &,- 83 &,$- 11 \mathrm{~ns}$ & $-1,23$ \\
\hline Ambicioso/Narcisista & ,15 ns & 1,64 &, $14 \mathrm{~ns}$ & 1,62 \\
\hline Leal/Dependiente & $36^{\star * *}$ & 4,29 & $32^{\star \star *}$ & 3,93 \\
\hline Impulsivo/Borderline &, $50^{\star \star *}$ & 6,14 & $45^{\star \star \star}$ & 5,82 \\
\hline Voluntarioso/Altruista & $31^{\star \star *}$ & 3,57 & $42^{\star \star \star}$ & 5,85 \\
\hline Crítico/Negativista & $64^{\star \star \star}$ & 9,29 & $60^{\star \star \star *}$ & 8,67 \\
\hline Pasivo/Depresivo & $.59^{\star \star \star}$ & 7,10 &, $50^{\star \star \star}$ & 6,78 \\
\hline Inseguro/Evitativo &, $41^{\star * *}$ & 4,98 & $42^{\star \star \star}$ & 5,30 \\
\hline
\end{tabular}

El segundo paso (línea b Fig. 2) consistió en el análisis de la relación entre cada una de las variables predictoras y cada una de las variables mediadoras (todos los EP medidos por el PSSI) (Tablas 9, 10 y 11).

Los tablas con los resultados de los análisis correspondientes al tercer y cuarto paso del modelo mediacional (línea $c$ y $d$ de la Fig. 2) no se presentan en este artículo por razones de espacio (para una revisión de los mismos ver Cordero, 2005).

A continuación se describen los resultados más relevantes en relación a cada una de las escalas afectivas del BEF-3 (Tabla 5).

Tristeza: En ambas muestras la relación entre frustración y tristeza fue mediada parcialmente por el EP pasivo (depresivo).
Muestra 1: La relación directa entre la frustración y el afecto negativo (tristeza) fue significativa $(\beta=.48, t(123)=6.11, p<.001)$. La frustración predice la aparición de afectos negativos. Cuando la variable frustración y el EP pasivo se introducen simultáneamente en los análisis, el EP pasivo predice la tristeza $(\beta=.59, t(123)$ $=6.87, p<.001)$. La relación directa entre frustración y tristeza desaparece cuando el EP pasivo es controlado $(\beta=-.11, t(123)=1.30, n s)$. El Test de Sobel fue significativo $(z=5.41$, $p<.001)$. El EP pasivo es un mediador parcial significativo para la relación entre frustración y tristeza. En otras palabras tras una frustración las personas con un estilo pasivo de personalidad tienen riesgo de desarrollar afectos negativos, tales como la tristeza. Esto es válido también para la muestra 2 (Fig. 4).

Fig. 3. Muestra 1. Análisis de mediación significativo con el EP depresivo como mediador de la relación entre frustración y tristeza.

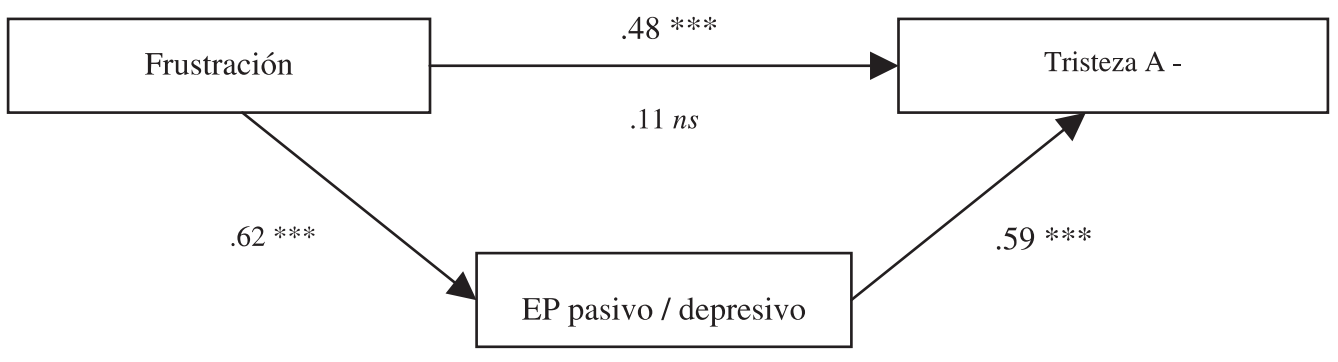


Fig. 4. Muestra 2. Análisis de mediación significativo con el EP depresivo como mediador de la relación entre frustración y tristeza. El resultado del Test de Sobel fue significativo $(z=4.04, p<001)$.

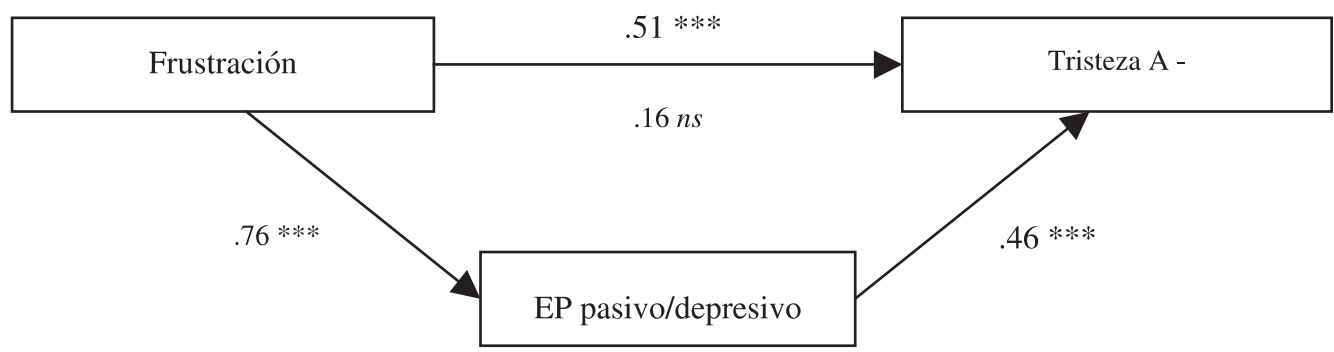

Excitación (en inglés "arousal", activación sensorial negativa): En ambas muestras la relación entre frustración y excitación fue mediada parcialmente por el EP pasivo (depresivo).

En contraste con lo esperado, ningún EP fue un mediador significativo para excitación y tristeza, cuando los predictores demandas y presión fueron introducidos en los análisis de regresión.

Disminución de la alegría (afecto positivo): Los resultados para esta reacción afectiva no se replican para ninguno de los tres predictores explorados.

Desánimo/Inhibición (inhibición del afecto positivo): El EP pasivo (depresivo) tiene una función mediadora parcial para esta reacción afectiva con las variables demandas y amena- zas como predictoras. A continuación se describen los resultados con la variable demandas como predictora.

Muestra 1: Como puede observarse en la tabla 7 la relación directa entre demandas y desánimo fue significativa $(\beta=.42, t(123)=5.14$, $p<.001)$. La variable demandas predice la aparición del desánimo (inhibición del afecto positivo). Cuando la variable demandas y el EP pasivo se introducen simultáneamente en los análisis, el EP pasivo predice el desánimo ( $\beta=.64$, $t(122)=7.70, p<.001)$. Posteriormente la relación directa entre demandas y desánimo desaparece cuando el EP pasivo es controlado ( $\beta$ $=.04, t(123)=.48, n s)$. El EP pasivo es un mediador parcial para la relación entre demandas y desánimo. Esto vale también para la muestra 2 (Fig. 6).

Fig. 5. Muestra 1. Análisis de mediación significativo con el EP pasivo (depresivo) como mediador de la relación entre demandas y desgano. El resultado del Test de Sobel fue significativo $(z=5.57, p<.001)$

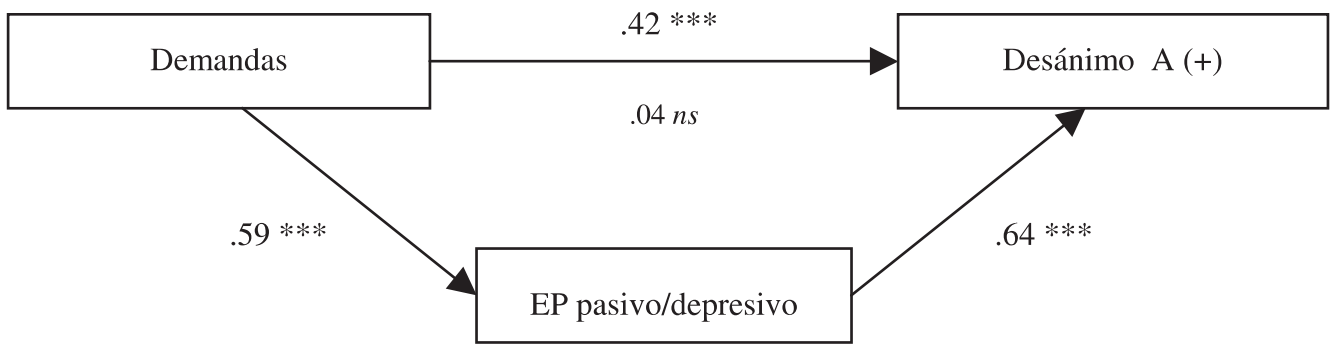

Fig. 6. Muestra 2. Análisis de mediación significativo con el EP pasivo (depresivo) como mediador de la relación entre demandas y desgano. El resultado del Test de Sobel fue significativo $(z=5.58, p<.001)$

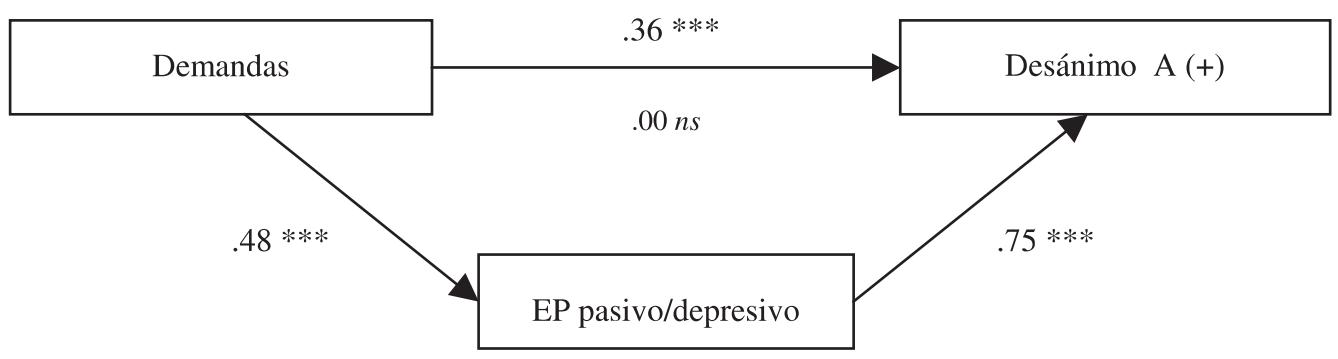


Por otra parte en ambas muestras el EP impulsivo (borderline) media la relación entre presión y desánimo.

Disminución de la activación motora: El EP pasivo (depresivo) es un mediador parcial para esta reacción afectiva con los tres predictores. Con la variable amenazas/presión como predictora tienen los siguientes EP una función mediadora parcial: inseguro (evitativo), impulsivo (borderline) y desconfiado (paranoide).

Disminución de la serenidad: La relación entre frustración y una disminución de la serenidad es mediada parcialmente en ambas muestras por el EP pasivo (depresivo) y el EP impulsivo (borderline). Ninguno de los EP explorados obtiene una función mediadora para esta reacción afectiva con las variables demandas y amenazas/presión como predictoras.

Irritabilidad: La relación entre frustración e irritabilidad es mediada parcialmente en ambas muestras por el EP pasivo (depresivo) y el EP impulsivo (borderline). Estos resultados se re- plican para los análisis de mediación con la variable presión como predictora, en donde además de los estilos mencionados, el EP crítico (negativista) también obtiene una función mediadora. En ambas muestras se impone el EP impulsivo (borderline) como el mediador más significativo ${ }^{5}$ para irritabilidad (Fig. 7 y 8 ).

Ningún EP es un mediador para la irritabilidad con la variable demandas como predictora.

Estilos de personalidad y autorregulación: Se realizaron análisis de regresión para explorar si la interacción entre cada uno de los EP (subescalas PSSI) y los déficits en las competencias autorregulatorias (orientación al estado prospectiva y tras un fracaso) tenía una función mediadora en la relación entre frustración y una serie de reacciones afectivas (BEF-3). Estos análisis no se calcularon para las variables predictoras demandas y amenazas, como en el resto de los análisis presentados hasta ahora.

Fig. 7. Muestra 1. Análisis de mediación significativo con el EP impulsivo (borderline) como mediador de la relación entre amenazas (presión) e irritabilidad. El resultado del Test de Sobel fue significativo $(z=4.18, p<.001)$

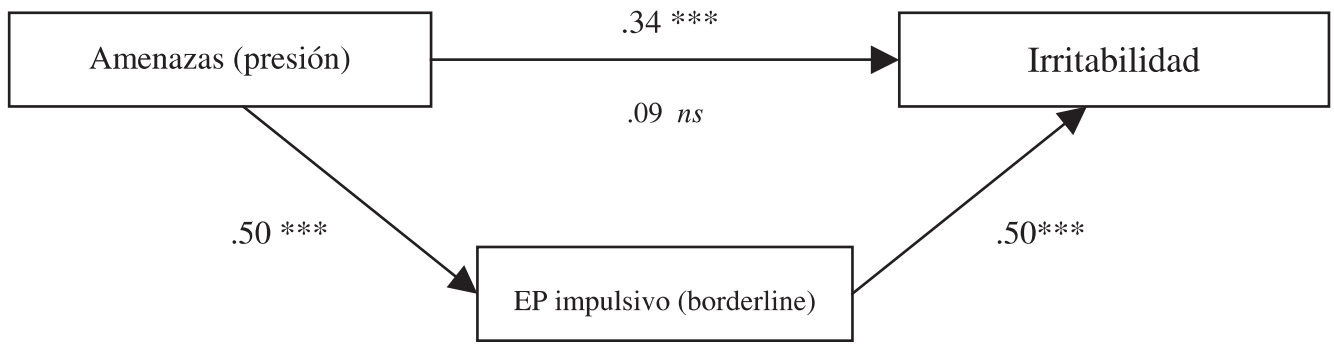

Fig. 8. Muestra 2. Análisis de mediación significativo con el EP impulsivo (borderline) como mediador de la relación entre amenazas (presión) e irritabilidad. El resultado del Test de Sobel fue significativo $(z=3.35, p<.001)$

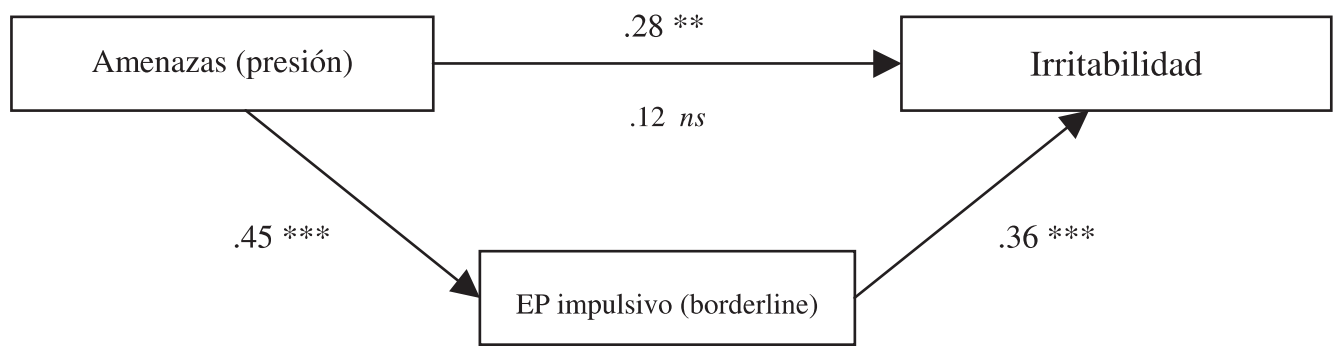

\footnotetext{
${ }^{5}$ Cuando más de un estilo de personalidad obtuvo un rol mediador para un afecto (variable dependiente), se introdujeron simultáneamente en los análisis de regresión (bloque 2). El objetivo fue investigar si alguno de los ellos tenía un rol mediador más importante para la relación específica estudiada.
} 
En contra de lo esperado, las combinaciones "poco favorables" entre cada uno de los EP y las variables de autorregulación (por ejemplo la combinación EP pasivo (depresivo) de la personalidad y orientación al estado tras un fracaso (OEF)) no obtuvieron en ningún caso un rol mediacional significativo para la relación entre frustración - reacciones afectivas negativas. Se esperaba que la orientación al estado tuviera una contribución adicional a la de los EP en la relación entre frustración - reacciones afectivas. En otro estudio (Cordero, 2007) se realizaron los mismos análisis utilizando como variables dependientes (criterio) una variedad de síntomas (medidos a través del SCL-90). Los resultados mostraron al igual que en este estudio que la interacción entre EP y OEF/OEP no media la relación entre frustración y síntomas psíquicos. Los hallazgos sí muestran que, al igual que en el estudio de Baumann, Kaschel y Kuhl (2005), los déficits en la autorregulación (OEF/OEP) moderan la relación entre los EP y un gran número de síntomas.

\section{Resumen y discusión}

El objetivo de esta investigación fue estudiar y comparar la función mediadora de catorce EP en la relación entre el estrés y/o la frustración de necesidades y el desarrollo de diversas reacciones afectivas. Los resultados de todos los análisis de mediación calculados arrojan los siguientes resultados a destacar:

- Los datos obtenidos apoyan la hipótesis de partida: la frustración de las necesidades básicas y el estrés tienen efectos negativos sobre el bienestar psicológico. Las variables frustración, demandas y amenazas predicen las mismas reacciones afectivas: tristeza, excitación, desánimo, irritabilidad y una reducción de la activación, la alegría y la serenidad (Tablas 6, 7 y 8 ). En otras palabras estas variables predicen el desarrollo de afecto negativo y la inhibición del afecto positivo.

- Globalmente los resultados obtenidos (Tabla 5) sugieren que los EP con una función mediadora parcial significativa para las relaciones exploradas son los siguientes: estilo pasivo (depresivo), espontáneo (borderline), crítico (negativista o pasivo/ agresivo) e inseguro (evitativo). Estos EP aparecen como factores de riesgo para el desarrollo de afecto negativo y/o inhibición del afecto positivo tras una frustración o bajo situaciones de estrés. De todos los EP anteriormente mencionados los principales mediadores son: estilo pasivo (depresivo), espontáneo (borderline) e inseguro (evitativo). Los resultados para este último $E P$, sin embargo, en general no se replican. Se destaca especialmente la función mediadora del estilo pasivo (depresivo).

- En contra de lo esperado (hipótesis 2) los siguientes EP no median ninguna de las relaciones exploradas: esmerado (obsesivo), idiosincrático (esquizotípico), reservado (esquizoide), leal (dependiente) y voluntarioso (altruista). Si en cambio se cumple la hipótesis 3: los EP con una baja sensibilidad para el afecto negativo y/o una alta sensibilidad para el afecto positivo (antisocial, narcisista, rapsódico e histriónico) no median ninguna de las relaciones exploradas.

- En consonancia con lo esperado (hipótesis 4), los EP con una función mediadora muestran un perfil diferente de acuerdo al predictor (por ejemplo: el estilo pasivo (depresivo) es un mediador para la disminución de la serenidad con las variables frustración y amenazas como predictoras, no así con la variable demandas). El resultado más importante en este sentido se observa en relación a los afectos negativos (tristeza y excitación). Como puede verse en la tabla 5 ningún EP (incluso aquellos que se impusieron como los mediadores más importantes: $\mathrm{DP}$ y $\mathrm{BL}$ ) media la relación para estos afectos cuando las variables predictoras son demandas y amenazas, si en cambio con la variable frustración como predictora.

- El EP pasivo (depresivo) media en ambas muestras la relación entre frustración y tristeza y entre frustración y excitación. Este estilo además es un mediador parcial para el desánimo (con las variables demandas y presión como predictoras) y para una reducción de la activación motora (con todos los predictores). Es decir que el EP pasivo (depresivo) reacciona prácticamente a todos los predictores con una inhibición del afecto positivo, mientras que solo a la frustración con afecto negativo. Este hallazgo apoya la afirmación de que las reacciones depresivas se asocian fuertemente con una inhibición de afecto positivo y que no necesariamente van acompañadas de un au- 
mento del afecto negativo (Watson \& Tellegen, 1985, Clark \& Watson, 1991).

- Con respecto al EP impulsivo (borderline) se destaca su rol mediador para la irritabilidad (con los predictores frustración y amenazas). Estos resultados apoyan la relación, frecuentemente reportada en la literatura clínica, entre el trastorno análogo a este estilo (trastorno borderline de la personalidad) y la irritabilidad.

- El estilo critico (negativista o pasivo/agresivo) media la relación entre presión e irritabilidad.

En líneas generales los resultados apoyan la hipótesis, derivada de los postulados de la Teoría PSI, de que los EP y las disposiciones afectivas que los subyacen juegan un papel importante en las relaciones investigadas y refuerzan la diferenciación entre estilos de personalidad, competencias de autorregulación y factores de estrés a lo largo de las dimensiones de afecto negativo y positivo.

Los hallazgos obtenidos sugieren que los predictores tienen efectos diferentes. En este sentido algunos datos sugieren que la variable demandas predice mejor la inhibición del afecto positivo (ej. desánimo) que el desarrollo de afecto negativo (ej. tristeza), en tanto los análisis con esta variable como predictora fueron significativos para el desánimo y una reducción de la activación motora (es decir inhibición del afecto positivo). No fueron, sin embargo, significativos para las escalas relacionadas con el afecto negativo. Esto concuerda con el postulado de la teoría PSI respecto a que el factor demandas se asocia con una disminución del afecto positivo más que con un aumento del afecto negativo (lo que correspondería al factor amenazas/ presión). Con respecto a la frustración, podría interpretarse que esta variable es más importante para el desarrollo de afectos negativos que el estrés. ¿Por qué reaccionan los EP con función mediadora (DP y BL) con tristeza y excitación a la frustración y no a la presión? Desde el punto de vista teórico era esperable que estos $E P$, que se caracterizan por una fuerte sensibilidad para el afecto negativo, reaccionaran a la presión con tristeza y excitación. Además, desde el punto de vista de la teoría PSI, este predictor (presión o amenazas) se acompaña de un aumento del afecto negativo.

Esta disociación en los resultados de las variables frustración y presión (amenazas) pue- de explicarse desde la teoría PSI del siguiente modo: podría pensarse que detrás de una frustración de las necesidades existe un "daño" más fundamental y profundo del self (es decir de nuestras necesidades esenciales) que en el caso de la presión (entendida como una vivencia de estrés más situacional y pasajera que la frustración). En efecto, la frustración de las necesidades básicas fue medida a través de una escala del MUT que representa una constelación permanente de los macrosistemas cognitivos, mientras que presión y demandas representan la percepción subjetiva de las circunstancias de vida actuales.

Por otra parte, el estilo pasivo (depresivo) se asocia, de acuerdo a la teoría PSI, simultáneamente con dos factores de riesgo: una fuerte sensibilidad para el afecto negativo y una baja sensibilidad para el afecto positivo [A- \& $A(+)]$. Esta combinación puede conducir simultáneamente a una inhibición volitiva y del self, lo que se corresponde con una dominancia del hemisferio izquierdo. Esta dominancia se relaciona con una rigidez en el pensamiento, que aumenta la experiencia de emociones negativas (Kuhl, 2001).

Los resultados sugieren la importancia de la afectividad negativa en las relaciones exploradas, en tanto de acuerdo a la teoría PSI los mediadores más importantes (DP y BL) tienen en común el que se relacionan con una fuerte sensibilidad a reaccionar con afecto negativo, afecto que, de acuerdo a la segunda hipótesis de modulación afectiva, inhibe el acceso al self y aumenta la orientación hacia las incongruencias y detalles aislados.

La teoría PSI se apoya en la neurobiología para explicar por qué la inhibición del self aumenta el riesgo de desarrollar síntomas psíquicos. El afecto negativo inhibe el acceso a los procesos del hemisferio derecho, que se encuentran, de acuerdo a la teoría, relacionados con los procesos del self. Los procesos del hemisferio derecho están más implicados en la regulación de los procesos emocionales que los del hemisferio izquierdo (ej. Dawson \& Schell, 1982; Wittling, 1990 citados en Kuhl, 2001) y sus déficits dificultan la elaboración de los afectos negativos, llevando a la utilización de estrategias ineficientes de afrontamiento (Schore, 1994, 1996 citado en Kuhl, 2001). Kuhl (2001) plantea como hipótesis que el hipocampo posee muchas de las funciones que se asocian en la teoría PSI 
con la memoria de extensión y el self. Las funciones cognitivas y reguladores del afecto del hipocampo pueden inhibirse por el estrés excesivo (y el correspondiente aumento de la concentración de cortisol), lo que psicológicamente supone una regresión a formas más elementales de procesamiento de la información y el temor (Metcalfe \& Jacobs, 1998; Sapolsky, 1992 citado en Kuhl, 2001).

Ahora bien una pregunta que se impone aquí es ¿por qué los otros EP con estas vulnerabilidades $[A-\& A(+)]$ no median las relaciones exploradas? Los análisis de correlaciones de Pearson en ambas muestras indican que la escala pasivo/depresiva correlaciona positiva y significativamente con un gran número de escalas del PSSI. Esto podría estar indicando que esta escala es la que mejor representa el componente depresivo (u otros aspectos) del resto de los EP. Otro aspecto importante a tener en cuenta, que se relaciona con el anterior, es si la escala pasivo/depresiva del PSSI evalúa un estilo de personalidad o si evalúa depresión. La separación empírica de los rasgos depresivos de la personalidad y la depresión clínica es muy difícil. Esta es una de las razones por la cual el trastorno depresivo de la personalidad no se encuentra en el DSM-IV, sino en estudio para su posible inclusión en futuras ediciones del DSM. En otra parte de esta investigación, que no se desarrolla en este artículo, se calculó el grado de correlación entre la escala pasivo/depresiva del PSSI y la escala de depresión del SCL-90. Los resultados muestran una correlación positiva y significativamente alta entre ambas escalas. En casi todos instrumentos que miden el la personalidad se encuentra un fuerte solapamiento con las medidas de los síntomas (Huprich, 2002). Por las razones explicitadas se considera que los resultados referidos a la escala pasivo/depresiva deben ser tomados con precaución.

En cuanto a las limitaciones de este trabajo consideramos que es importante tener en cuenta la heterogeneidad de ambas muestras, que se refleja en las comparaciones de medias. Otro aspecto que podría verse como una limitación, son las medidas utilizadas para las variables frustración y de los factores de estrés, estrechamente vinculadas a la teoría PSI. Son necesarios otros estudios para replicar los resultados utilizando otros instrumentos de evaluación de la personalidad así como de la frustración y el estrés.

\section{REFERENCIAS}

Aiken, L. S., \& West, S. G. (1991). Multiple regression: Testing and interpreting interactions. Newbury Park, CA: Sage.

American Psychiatric Association (1994). Diagnostic and statistical manual of mental disorders (4th ed.: revised). Washington, DC: American Psychiatric Association.

Baron, R. M., \& Kenny, D. A. (1986). The moderatormediator variable distinction in social psychological research: Conceptual, strategic, and statistical considerations. Journal of Personality and Social Psychology, 51, 11731182.

Baumann, N. (1998). Selbst- versus Fremdbestimmung: Zum Einfluss von Stimmung, Bewusstheit und Persönlichkeit. [Determinación propia o ajena: hacia la influencia del ánimo, la conciencia y la personalidad]. Doctorado no publicado. Universität Osnabrück.

Baumann, N., \& Kuhl, J. (2002). Intuition, affect, and personality: Unconscious coherence judgments and self-regulation of negative affect. Journal of Personality and Social Psychology, 83, 12131223.

Baumann, N., Kaschel, R., \& Kuhl, J. (2005). Striving for unwanted goals: stress-dependent discrepancies between explicit and implicit achievement motives reduce subjective wellbeing and increase psychosomatic symptoms. Journal of Personality and Social Psychology, 89(5):781-99

Baumann, N., Kaschel, R., \& K. Kuhl, J. (2004). From Personality Styles to Symptom Formation: The Moderating Role of Self-Regulation. Manuscrito enviado. Universität Osnabrück

Biebrich, R. \& Kuhl, J. (2002a). Neurotizismus und Kretivität: Strukturelle Unterschiede in der Beeinflussung kreativer Leistung. [Neuroticismo y creatividad: la influencia de las diferencias estructurales en el rendimiento creativo]. Zeitschrift für Differentielle und Diagnostische Psychologie, 23, 171-190.

Biebrich, R. \& Kuhl, J. (2002b). Selbststeuerung und affektive Sensibilität: Persönlichkeitsspezifische Antezedenzien der Depressivität. [Autorregulación y sensibilidad afectiva: antecedentes de personalidad para la depresión]. Zeitschrift für Psychologie, 210, 74-86.

Brunstein, J.C. (2001). Persönliche Ziele und Handlungs- versus Lageorientierung: Wer bindet sich an realistische und bedürfniskongruente Ziele? [Personal goals and action versus state orientation: Who builds a commitment to realistic and need-congruent goals?]. Zeitschrift für Differenzielle und Diagnostische Psychologie, 22, 1-12.

Clark, L.A. \& Watson, D. (1999). Temperament: a new paradigm for trait psychology. En 
Pervin,L.A., y John, O.P. (Eds.). Handbook of personality: theory and research. The Guilford Press: New York.

Cordero, S. (2005). Persönlichkeitsstile und psychische Erkrankung (Achse I und II): Zur Rolle von Bedürfnisfrustration, Stress, Affekten und Selbststeuerungsdefiziten. [Estilos de personalidad y enfermedades psíquicas (Eje I y II): el rol de la frustración, el estrés, los afectos y los déficits en el autogobierno]. Tesis de doctorado. Universidad de Osnabrück. Publicación electrónica en la Biblioteca de la Universidad de Osnabrück (http://elib.ub.uni-osnabrueck.de/ publications/diss/E-Diss489.thesis.pdf) y en los catálogos de bibliotecas alemanas (http://dbfopac.ddb.de)

Cordero, S. (2007). Estrés/frustración y enfermedades psíquicas (Eje I): el rol mediador de los estilos de personalidad. Revista de la Sociedad de Psiquiatría Biológica del Uruguay.

Dieffendorf, J, Hall, R., Lord R.. \& Strean, M. (2000). Action-State Orientation: Construct Validity of a Revised Measure and Its Relationship to WorkRelated Variables. Journal of Applied Psychology, 85, 250-263.

Emmons, R. A. (1986). Personal strivings: An approach to personality and subjective well-being. Journal of Personality and Social Psychology, 51, 1058-68.

Eysenck, H. J. (1990). Biological dimensions of personality. In L. A. Pervin (Ed.), Handbook of personality: Theory and research (pp. 244-276). New York: Guilford.

Gray, J.A. (1981). A critique of Eysenck's theory of personality. En H. J. Eysenck (Ed.), A model of personality (pp. 246-276). Nueva York: Springer.

Gray, J. A. (1987). The psychology of fear and stress ( $2^{\text {nd }}$ ed.). Cambridge: University Press.

Greenwald, A. G., \& Banaji, M. R. (1995). Implicit social cognition: Attitudes, self-esteem, and stereotypes. Psychological Review, 102, 4-27.

Hautzinger, M. (1994). Action control in context of psychopatological disorders. En J. Kuhl \& Beckmann (Hrsg.). Volition and Personality: Action versus state orientation (Pag. 209-215). Seattle: Hogrefe.

Huprich, S.K., Sanford, K. \& Smith, M. (2002). Psychometric Evaluation of the Depressive Personality Disorder Inventory. Journal of Personality Disorders. 16(3), 255-269

Holmes, T. H., \& Rahe, R. H. (1967). The social readjustment rating scale. Journal of Psychosomatic Research, 11, 213-218.

Jung, C. G. (1936/1990). Typologie [Typologia]. München, Germany: dtv.

Koole, S., \& Jostmann, N. (2003). Getting a grip on your feelings: Effects of action orientation and social demand on intuitive affect regulation. Manuscript submitted for publication. Fee University of Amsterdam.
Kuhl, J. (1983). Motivation, Konflikt und Handlungskontrolle. [Motivación, conflicto y control de la acción]. Heidelberg: Springer-Verlag.

Kuhl, J. (1994). Action versus state orientation: Psychometric properties of the Action-Contol-

Scale (ACS-90). En J. Kuhl \& J. Beckmann (Eds.), Volition and personality: Action versus state orientation (pp. 47-59). Göttingen, Germany: Hogrefe.

Kuhl, J. (1995). Introjektion, Alienation und Grübeln: Von rationalen Motivationsmodellen zu EGG Korrelaten volitionaler Hemmung. [Introjección, alienación y rumiación: de los modelos de motivación racionales a los correlatos del EGG de la inhibición volitiva]. Manuscrito no publicado. Universidad de Osnabrück.

Kuhl, J. (1998). Wille und Persönlichkeit: Von der Funktionsanalyse zur Aktivierungsdynamik psychischer Systeme. [Volición y Personalidad: el análisis funcional de la activación dinámica de los sistemas psíquicos]. Psychologische Rundschau, 49, 61-77.

Kuhl, J. (1999). Der Motiv-Umsetzung-Test (MUT). Test de Implementación de Motivos. Cuestionario no publicado. Universidad de Osnabrück.

Kuhl, J. (2000a). A functional-design approach to motivation and volition: The dynamics of personality systems interactions. En M. Boekaerts, P. R. Pintrich \& M. Zeidner (Eds.), Self-regulation: Directions and challenges for future research (pp. 111-169). New York: Academic Press.

Kuhl, J. (2001). Motivation und Persönlichkeit: Interaktionen psychischer Systeme [Motivation and personality: Interactions of mental systems]. Göttingen, Germany: Hogrefe.

Kuhl, J., \& Baumann, N. (2000b). Self-regulation and rumination: Negative affect and impaired selfaccessibility. En W. Perrig \& A. Grob (Eds.), Control of human behavior mental processes and consciousness: Essays in honor of the $60^{\text {th }}$ birthday of August Flammer (pp. 283-305). Mahwah, NJ: Erlbaum.

Kuhl, J. \& Beckmann, J. (1994). Volition and personality: Action versus state orientation. Göttingen/Seattle: Hogrefe.

Kuhl, J. \& Fuhrmann, A. (1998). Decomposing selfregulation and self-control: The volitional components checklist. En J. Heckhausen \& C. Dweck (Eds.), Life span perspectives on motivation and control (pp. 15-49). Mahwah, NJ: Erlbaum.

Kuhl, J., \& Kazén, M. (1997). Das PersönlichkeitsStil-und-Störungs-Inventar (PSSI): Manual [The Personality-Styles-and-Disorder-Inventory (PSSI): Manual]. Göttingen, Germany: Hogrefe.

Kuhl, J., \& Kazén, M. (1999). Volitional facilitation of difficult intentions: Joint activation of intention memory and positive affect removes stroop interference. Journal of Experimental Psychology: General, 128, 382-399. 
Linville, P.W. (1987). Self-complexity as a cognitive buffer against stress-related illness and depression. Journal of Personality and Social Psychology, 52, 663-676.

Millon, T. \& Everly, G. (1985). Personality and disorders. New York: Wiley.

Murray, H.A. (1943). Thematic Apperceptive Test Manual. Cambridge: Harvard University Press.

Oldham, J.M. (Hrsg.) (1990). Personality disorders: New perspectives on diagnostic validity.

Washington, DC: American Psychiatric Press.

Ramírez, Esteve y López (2001). Neuroticismo, afrontamiento y dolor crónico. Anales de psicología. Vol.17. 1, 129-137

Ryder, A. G., \& Bagby, R.M. (1999). Diagnostic viability of depressive personality disorder: Theoretical and conceptual issues. Journal of Personality Disorders, 13, 99- 117.

Sánchez Cánovas, J. y Sánchez López, M.P. (1994). Psicología diferencial: Diversidad e individualidad humanas. Madrid:Ramón Areces.

Schmitz, B. Schuhler, P., Handke-Raubach, A., Jung, A. (2001) Kognitive Verhaltenstherapie bei Persönlichkeitsstörungen und unflexiblen Persönlichkeitsstilen. Ein psychoedukativ- und kompetenzorientiertes Therapieprogramm [Terapia cognitivo-comportamental para los trastorno de la personalidad y estilos de personalidad inflexibles. Un programa de terapia orientado a la psicoeducación]. Pabst Science Publishers

Scully, J. A., Tosi, H., \& Banning, K. (2000). Life event checklists: Revisiting the social readjustment rating scale after 30 years. Educational and Psychological Measurement, 60, 864-876.

Showers, C.J. \& Kling, K.C. (1996). Organization of self-knowledge: Implications for recovery from sad mood. Journal of Personality and Social Psychology, 70, 578-590.

Sobel, M. (1982). Asymptotic confidence intervals for indirect effects in structural equations

models. In S. Lei hart (Ed), Sociological methodology (pp. 290-312).San Francisco: Jossey-Bass.

Watson, D. \& Tellegen, A. (1985). Toward a consensual structure of mood. Psychological Bulletin, 98, 219-235.

Watson, D., Clark, L. A., \& Tellegen, A. (1988). Development and validation of brief measures of positive and negative affect: The PANAS scales. Journal of Personality and Social Psychology, 54, 1063-1070. 